\title{
Hedging the risk of delayed data in defaultable markets
}

\author{
Ramin Okhrati*
}

\begin{abstract}
We investigate hedging the risk of delayed data in certain defaultable securities through the local risk minimization approach. From a financial point of view, this indicates that in addition to the risk of default, investors also face incomplete accounting data. In our analysis, the delay is modeled by a random time change, and different levels of information, including the full market's, management's, and investors' information, are distinguished. We obtain semi-explicit solutions for pseudo locally risk minimizing hedging strategies from the perspective of investors where the results are presented according to the solutions of partial differential equations. In obtaining the main results of this paper, we apply a filtration expansion theorem that determines the canonical decomposition of stopped special semimartingales in an enlarged filtration of investors' information.
\end{abstract}

Keywords: Defaultable claims, Pseudo local risk minimization, Intensity, Delayed data, Random time change

*University of Southampton, Southampton, UK, Email: r.okhrati@soton.ac.uk 


\section{Introduction}

There is an asymmetry of access to data between the management board of a company and investors. The data available to investors is normally lagged, for instance, they are informed of the company's asset values at some point in future. The problem gets even more complicated in the context of credit derivatives where in addition to this asymmetry of information, investors also face the risk of default. We analyze hedging the risk of defaultable claims in a partial information credit risk modeling where it is assumed that investors receive company's asset values with some delays. In order to do this, first, we need to model and measure the arrival rate of default.

The concept of intensity (or stochastic intensity) is a popular approach to model the arrival rate of default. There are two notions of intensity. The first one is defined through a hazard-based approach in which the hazard process of a default time is assumed to be absolutely continuous with respect to the Lebesgue measure. Then the RadonNikodym derivative of the hazard process with respect to the Lebesgue measure is called the intensity (or hazard rate) process of the default time, see Chapter 5, in particular Section 5.1.4, of [6].

The second notion is based on Doob-Meyer's decomposition, where the intensity process of the default time $\tau$ is defined as the Radon-Nikodym derivative (if it exists) of the compensator of the default indicator process, $\left(1_{\{\tau \leq t\}}\right)_{t \geq 0}$, in an appropriate filtration. Proposition 5.1.3 of [6] (see also Section 5.1.4 of [6]) shows that an intensity process in the sense of the first definition, is also an intensity process (once restricted up to the default time) in the sense of the second one, but the other way around does not always hold.

Therefore, because of its flexibility, we choose the second notion of intensity to model the default rate. Note that the existence of the intensity process of a default time is not always guaranteed, see Proposition 6.1 of [14]. For interesting examples, discussions, and a general procedure of constructing intensity based on the second notation, we refer to [16].

Another reason to choose the second notion of intensity is concerning the continuously delayed filtration in [15] that we apply to model the delay in data and the investors' information. The existence of the intensity process of a default time and its closed-form formula based on the second notion, are guaranteed and well investigated in certain models under the continuously delayed filtration, see Theorem 3.1 of [15]. This theorem is used to obtain a semi-closed-form formula in Example 4.2 which is one of the important 
cases that we study.

The next step is to choose an appropriate risk management approach. The presence of the risk of default and the partial information would lead to an incomplete market; hence, we should use a risk management method that is applicable to incomplete markets. This is done through the local risk minimization (LRM) approach that we use to identify hedging strategies of defaultable claims.

The LRM approach has already been applied in managing the risk of defaultable markets, for example in [3], [4], and [5]. One of the advantages of this method is its mathematical flexibility. In [28], under some conditions (where square integrability is one of the important ones) on the underlying process that are reviewed in Section 2.1, it is shown that finding the hedging strategy of a defaultable claim in the LRM framework is equivalent to finding the so called Pseudo-Locally Risk Minimizing (PLRM) hedging strategy which in turn is determined through a special orthogonal decomposition of the claim known as Föllmer-Schweizer (FS) decomposition. Therefore, we focus on PLRM hedging strategies, and given a defaultable claim, the aim is to obtain its FS decomposition.

Assuming the existence of a hazard rate process (the first notation of intensity discussed earlier) and the full observation of the underlying assets, modeled by diffusion processes, the FS decompositions and hence the PLRM hedging strategies of defaultable claims are discussed in [3], [4], and [5]. In these works, modeling the risk of default by the first notion of intensity, plays an important role as it allows to use the results of Chapter 5 of $[6]$.

Normally, in defaultable markets, the default time is not necessarily measurable with respect to the investors' information, so the LRM or the PLRM approach is carried out in an enlarged filtration of the investors' data to turn the default time into a stopping time. In simple terms, one starts with a reference filtration containing the information from non-defaultable markets observable to investors, and then this filtration is expanded to a new one in which the default time is a stopping time.

It is important to note that a semimartingale in a reference filtration does not preserve its properties once the filtration is expanded, see [19]. In [5], it is assumed that hedging stops after default which enables them to dismiss the H-hypothesis (this hypothesis holds if any martingale in the reference filtration remains a martingale in the expanded one). In addition, they investigate the recovery of defaultable claims assuming dependency between the default time and underlying assets. 
One can also assume that the default time is independent of underlying assets. This is less favored (at least in our model) in finance as there could be correlation between the default time and underlying assets. In [8] and [9], it is assumed that the information from underlying assets is contained in the investors' filtration where the hedging analysis is carried out.

We challenge the above assumptions by presenting a credit risk model in which investors cannot fully observe underlying assets. In what follows, we summarize the main contributions of our work more specifically.

We obtain semi-closed-form formulas for PLRM hedging strategies of defaultable claims under the continuously delayed filtration. Under some conditions, the final formulas are numerically implementable, and we distinguish the full market's, managers', and investors' information. The default time may or may not be adaptable with respect to neither the investors' nor the managers' filtration, and it could have correlation with underlying assets (which are modeled by continuous special semimartingales) and hence the information available to investors.

In our work, the default rate can be modeled through either notions of stochastic intensity. This allows to analyze models where the default time is defined economically and endogenously through the first hitting time of a firm's assets (for which investors might not be informed instantly) to a barrier. Normally, in hazard rate approaches to obtain hedging strategies, e.g. in [3] and [4], it is not economically clear why default occurs; in other words, the nature of default in terms of asset and liabilities cannot be explained.

In order to derive the hedging strategy of a defaultable claim, we obtain its FS decomposition in an enlarged filtration of investors. Although there are general results that guarantee the existence of the FS decomposition even under different levels of information, see [8], [10], and [13], obtaining numerically implementable results remains a challenge. Normally, the main method of finding the FS decomposition is by transferring the original probability measure to a minimal equivalent local martingale measure (MELMM), for instance as discussed in [8], [11], and [28].

We obtain semi-closed-form solutions of the PLRM hedging strategies without altering the underlying probability measure and without applying the MELMM method. We achieve this through applying an enlargement filtration theorem. Then canonical and Kunita-Watanabe (KW) decompositions are used to represent the PLRM hedging strategies through solutions of partial differential equations (PDEs) which are numeri- 
cally implementable. The same idea is applied in [24] in the context of structural models using partial-integro differential equations and finite variation Lévy processes to model underlying assets.

To the best of our knowledge this is the first time that hedging the risk of delayed data in defaultable markets is tackled; in particular, under these circumstances.

The paper is outlined as follows. In Section 2, we explain the model and some preliminaries; in particular, a brief review of the LRM approach is provided in Section 2.1. Canonical and KW decompositions of certain special semimartingales are discussed in Section 3 which are required to carry out our analysis. Section 4 is devoted to some applications, examples, and finding the FS decompositions and hence the PLRM hedging strategies. Finally, we conclude the paper in Section 5. In Section 7.1, some technical results are reviewed for unfamiliar readers.

\section{The Model Setup}

We suppose that all the random variables and stochastic processes in our model are defined on $(\Omega, \mathfrak{F}, \mathscr{F}, \mathbb{P})$ which is a complete filtered probability space satisfying the usual hypotheses where $\mathscr{F}=\left(\mathscr{F}_{t}\right)_{t \geq 0}$ represents the flow of information from all markets, including defaultable and non-defaultable ones, and $\mathscr{F}_{t} \subset \mathfrak{F}$ for all $t \geq 0$. Since we deal with more than one filtration in our work, henceforth, it is assumed that all filtrations applied in this paper satisfy the usual hypotheses.

In our framework, a level of information such as the investors' or management's information is modeled through a filtration. For instance, the evolution of asset values of a company could be modeled by a stochastic process, then the natural filtration generated by this process can form a level of information that could be observable to either investors or the management. The next assumption models the management's information.

Assumption 2.1. The available information to the management board of a company at time $t \geq 0$ on $(\Omega, \mathfrak{F}, \mathbb{P})$ is modeled by a filtration $\mathscr{F}^{\mathscr{M}}=\left(\mathscr{F}_{t}^{\mathscr{M}}\right)_{t \geq 0}$ such that $\mathscr{F}_{t}^{\mathscr{M}} \subset \mathscr{F}_{t}$, for all $t \geq 0$.

Now, we specify stochastic processes that are used to model risky asset values. It is assumed that the default-free market is composed of two tradable assets: a risky asset, modeled by a stochastic process $X$, and a risk-free money market account. The evolution of the risky asset (for instance the company's asset values) is observable to the management, and hence $X$ is an $\mathscr{F}^{\mathscr{M}}$-adapted process. Furthermore, we assume that 
- The stochastic process $X=\left(X_{t}\right)_{t \geq 0}, X_{t}: \Omega \longmapsto(-\infty, \infty)$ is a continuous semimartingale (hence a special semimartingale) on $(\Omega, \mathfrak{F}, \mathscr{F} \mathscr{M}, \mathbb{P})$ admitting the canonical decomposition $X=X_{0}+M+A$, where $X_{0}$ is a constant, $M$ with $M_{0}=0$, is an $\mathscr{F}^{\mathscr{M}}$-local martingale, and $A$ with $A_{0}=0$, is $\mathscr{F}^{\mathscr{M}}$-predictable and of finite variation. Furthermore, the processes $A$ and the quadratic variation of $X$ (denoted by $[X, X]$ or $[X])$ are absolutely continuous ${ }^{1}$ with respect to the Lebesgue measure, i.e. for all $t \geq 0$,

$$
A_{t}=\int_{0}^{t} a_{s} d s, \text { and }[X]_{t}=\int_{0}^{t} b_{s} d s,
$$

where $a=\left(a_{t}\right)_{t \geq 0}$ and $b=\left(b_{t}\right)_{t \geq 0}$ are $\mathscr{F}^{\mathscr{M}}$-adapted càdlàg processes.

- The money market account (that is observable and measurable to both managers and investors) has a constant value of one at all times. This means that $X$ represents the discounted asset values.

The processes $A$ and $[X]$ are $\mathscr{F}^{\mathscr{M}}$-predictable, so without any loss in generality, we can assume that $a$ and $b$ are $\mathscr{F}^{\mathscr{M}}$-predictable too, see Proposition I.3.13 of [18]. In fact, for each $t \geq 0, \int_{0}^{t} a_{s} d s=\int_{0}^{t} a_{s-} d s, \mathbb{P}$-a.s., and $\left(a_{s-}\right)_{s \geq 0}$ is càglàd and hence predictable.

We suppose that investors are not immediately informed of the true values of the underlying asset, and they receive information with some delay modeled by a random time change $\alpha=\left(\alpha_{t}\right)_{t \geq 0}$. For example, if there is a constant delay $l>0$ in receiving the data, then $\alpha_{t}=(t-l)^{+}=\max (t-l, 0)$, for all $t \geq 0$. We formally define the random time change $\alpha$ in our work as follows.

Definition 2.1. The set $\alpha=\left\{\alpha_{t} ; t \geq 0\right\}$ on $(\Omega, \mathfrak{F}, \mathscr{F} \mathscr{M}, \mathbb{P})$ is called a time change if

1. $\alpha_{0}=0$,

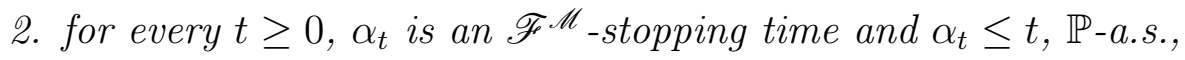

3. for every $\omega \in \Omega$, the sample path $t \longmapsto \alpha_{t}(\omega), t \geq 0$, is continuous and nondecreasing.

Remark 2.1. Note that in the literature such as Section 2 of Chapter VI of [12] or in [21], the random time change $\alpha$ is defined as any right-continuous non-decreasing family of $\mathscr{F}^{\mathscr{M}}$-stopping times. So in this sense, it would be better to call $\alpha$ in Definition 2.1 a

\footnotetext{
${ }^{1}$ Since $X$ is continuous, Lemma I.4.24 and Theorem I.4.47 of [18] show that $M, A$, and $[X]$ are continuous as well. However this does not imply that $A$ and $[X]$ are absolutely continuous with respect to the Lebesgue measure.
} 
continuous delayed time change; however, with some abuse of terminology, we still call it just a time change. Point (2) of the previous definition indicates that the delay is observable to the management, and if for all $t \geq 0$, we have $\alpha_{t}=t$, then the information is up to date. Furthermore, in order to guarantee that the time-changed processes remain continuous, point (3) additionally imposes the continuity assumption.

It is reasonable to assume that at some point, the management's information is disclosed to investors; hence, the investors' information can be modeled through a random time change of the management's information. Therefore, we can have the following definition.

Assumption 2.2. The information available to investors is denoted by $\mathscr{F}^{\mathcal{I}}$ and modeled by $\mathscr{F}_{t}^{\mathcal{I}}=\mathscr{F}_{\alpha_{t}}^{\mathscr{M}}, t \geq 0$. As usual, $\mathscr{F}_{\infty}^{\mathcal{I}}$ is the sigma-algebra generated by all $\mathscr{F}_{t}^{\mathcal{I}}, t \geq 0$. Since investors get their data through the random time change $\alpha$, instead of $X_{t}, t \geq 0$, we assume that they observe $Y_{t}=X_{\alpha_{t}}, t \geq 0$.

Remark 2.2. In [15], the filtration $\mathscr{F}^{\mathcal{I}}=\left(\mathscr{F}_{t}^{\mathcal{I}}\right)_{t \geq 0}$ is called the continuously delayed filtration.

Lemma 2.1. The process $Y=X_{\alpha}=\left(X_{\alpha_{t}}\right)_{t \geq 0}$ is a continuous process on $\left(\Omega, \mathfrak{F}, \mathscr{F}^{\mathcal{I}}, \mathbb{P}\right)$ admitting the canonical decomposition $Y_{t}=Y_{0}+A_{\alpha_{t}}+M_{\alpha_{t}}, t \geq 0$, where $M_{\alpha}, M_{0}=0$, is a continuous $\mathscr{F}^{\mathcal{I}}$-local martingale; $A_{\alpha}, A_{0}=0$, is a continuous $\mathscr{F}^{\mathcal{I}}$-adapted (hence $\mathscr{F}^{\mathcal{I}}$-predictable) process and of finite variation.

Proof. Continuity of $Y, A_{\alpha}$, and $M_{\alpha}$ follows from the continuity of $\alpha, X, A$, and $M$. Proposition V.1.5 of [26] shows that $M_{\alpha}$ is an $\mathscr{F}^{\mathcal{I}}$-local martingale. Since $\alpha$ is continuous and of finite variation, then $A_{\alpha}$ is $\mathscr{F}^{\mathcal{I}}$-predictable and of finite variation as well. Corollary I.3.16 of [18] shows that $Y=Y_{0}+A_{\alpha}+M_{\alpha}$ is the unique canonical decomposition of $Y$ in $\mathscr{F}^{\mathcal{I}}$.

Next, we explain the defaultable market model in our framework. In simple terms, a defaultable claim is a contingent derivative whose payoff is linked to a default event. More precisely, a default event on $(\Omega, \mathfrak{F}, \mathscr{F}, \mathbb{P})$ is modeled by an $\mathscr{F}$-stopping time $\tau$ : $\Omega \longmapsto[0, \infty)$ such that $\tau>0, \mathbb{P}$-a.s. and $\mathbb{P}(\tau>t)>0$ for all $t>0$. Recall that $\mathscr{F}$ represents the full information. A defaultable claim is then modeled by a triplet $(H, \tau, T)$ explained as follows: the owner of this claim receives $H \in L^{2}\left(\Omega, \mathscr{F}_{T}^{\mathcal{I}}, \mathbb{P}\right)$ at the maturity time $0<T<\infty$ if there is no default prior $T$ and zero otherwise, i.e. the payoff is equal to $H 1_{\{\tau>T\}}$. 
Remark 2.3. It is important to note that $\tau$ is an $\mathscr{F}$-stopping time, but it may not be an $\mathscr{F}^{\mathscr{M}}$ or $\mathscr{F}^{\mathcal{I}}$-stopping time which means that default might not be completely measurable at the management or investor level. Note that the case of recovery is not considered, and since the investors should be aware of the value of $H$ at $T, H$ is $\mathscr{F}_{T}^{\mathcal{I}}$ measurable. The latter indicates that unlike investors, the management might be informed of $H$ at or an earlier time than $T$. This makes sense financially as it reflects the fact that the management would have access to a more up to date data that can reveal the true value of $H$ earlier than the maturity.

Furthermore, we assume that the market models, from the perspective of either the investors or managers, are arbitrage free.

Although default might occur as a surprise to both investors and managers, it makes sense to assume that they are both informed of the occurrence of default in markets. This leads to the following definition.

Definition 2.2. The default indicator process $N=\left(N_{t}\right)_{t \geq 0}$ is defined by $N_{t}=1_{\{\tau \leq t\}}$, $t \geq 0$, and the filtration generated by $N$ is denoted by $\mathcal{N}=\left(\mathcal{N}_{t}\right)_{t \geq 0}$, i.e. $\mathcal{N}_{t}=\sigma\left(N_{u} ; u \leq\right.$ $t), \quad t \geq 0$.

There are other equivalent forms of $\mathcal{N}$, for instance, it can be shown that for all $t \geq 0, \mathcal{N}_{t}=\sigma(t \wedge \tau) \vee(\{\tau>t\})$, where $t \wedge \tau=\min (t, \tau)$ and $\sigma(t \wedge \tau)$ is the sigmaalgebra generated by the random variable $t \wedge \tau$, see Section 4.1 of [6]. Furthermore, since by Theorem 25, Chapter I of [25], $\mathcal{N}$ is a right continuous filtration, we also have $\mathcal{N}_{t}=\sigma(t \wedge \tau), t \geq 0$. From a financial point of view, under $\mathcal{N}$ at each time $t$, the agents or investors can observe the occurrence of default.

So far, we have defined four filtrations in our model: $\mathscr{F}, \mathscr{F}^{\mathscr{M}}, \mathscr{F}^{\mathcal{I}}$, and $\mathcal{N}$. The information available to investors comes from $\mathscr{F}^{\mathcal{I}}$ and $\mathcal{N}$. Since $\tau$ is not necessarily $\mathscr{F}^{\mathcal{I}}$ or $\mathscr{F}^{\mathscr{M}}$ measurable, any proper analysis from the investors' perspective requires an enlargement of $\mathscr{F}^{\mathcal{I}}$ that turns $\tau$ into a stopping time.

Concerning a filtration enlargement, there are two popular choices in finance. The first is a progressive filtration expansion of $\mathscr{F}^{\mathcal{I}}$ by $\tau$ defined by

$$
\left(\mathscr{F}_{t}^{\mathcal{I}} \vee \mathcal{N}_{t}\right)_{t \geq 0}
$$

On the other hand, it is easy to prove that for any $t \geq 0$, the set

$$
\left\{E \in \mathscr{F}_{\infty}^{\mathcal{I}} \vee \sigma(\tau): \quad E \cap\{\tau>t\}=E_{t} \cap\{\tau>t\}, \text { for some } E_{t} \in \mathscr{F}_{t}^{\mathcal{I}}\right\}
$$


is a sigma-algebra. The second notion of filtration expansion of $\mathscr{F}^{\mathcal{I}}$ by $\tau$ is defined by

$$
\left(\left\{E \in \mathscr{F}_{\infty}^{\mathcal{I}} \vee \sigma(\tau): E \cap\{\tau>t\}=E_{t} \cap\{\tau>t\} \text {, for some } E_{t} \in \mathscr{F}_{t}^{\mathcal{I}}\right\}\right)_{t \geq 0} .
$$

For more details of these filtration expansions and their applications, we refer to [19] and [20]. Comparing these two filtration expansions, the former is the favorite one as the definition of the latter implies the inclusion of partial information from $\mathscr{F}^{\mathcal{I}}$ up to infinity (and hence the anticipation of the future) which is not consistent from a financial perspective, see the discussions of Section 2.1 of $[16]^{2}$. Also, in [16], a more general type of filtration expansion is defined as follows.

Definition 2.3. The filtration $\mathscr{F}^{\mathcal{I}, \tau}$ is defined to be any filtration expansion of $\mathscr{F}^{\mathcal{I}}$ and $\mathcal{N}$ that turns $\tau$ into a stopping time, and for all $t \geq 0$, it satisfies

$$
\mathscr{F}_{t}^{\mathcal{I}, \tau} \cap\{\tau>t\}=\mathscr{F}_{t}^{\mathcal{I}} \cap\{\tau>t\}
$$

where $\mathscr{F}_{t}^{\mathcal{I}, \tau} \cap\{\tau>t\}=\left\{E \cap\{\tau>t\}: E \in \mathscr{F}_{t}^{\mathcal{I}, \tau}\right\}$, and a similar definition applies to $\mathscr{F}_{t}^{\mathcal{I}} \cap\{\tau>t\}$.

In Section 2.1 of [16], it is shown that both types of the filtration expansions (2) and (3) satisfy (4), and hence they are special cases. Next, we focus on modeling the arrival rate of default which can be done using two approaches.

Definition 2.4. Suppose that the hazard process $\Gamma=\left(\Gamma_{t}\right)_{t \geq 0}, \Gamma_{t}=-\ln \left(\mathbb{P}\left(\tau>t \mid \mathscr{F}_{t}^{\mathcal{I}}\right)\right)$, is well-defined for all $t \geq 0$. Assume that $\Gamma$ is absolutely continuous with respect to the Lebesgue measure with a non-negative Radon-Nikodym derivative $\lambda=\left(\lambda_{t}\right)_{t \geq 0}$ that is an $\mathscr{F}^{\mathcal{I}}$-adapted càdlàg process. The process $\lambda$ is called the intensity process of $\tau$. It is also referred to as the stochastic intensity (or the hazard rate) process.

On the other hand, the process $N=\left(1_{\{\tau \leq t\}}\right)_{t \geq 0}$ is an $\mathscr{F}^{\mathcal{I}, \tau}$-submartingale of class (D) which means that the set $\left\{N_{\mathcal{S}}\right.$ : for finite valued $\mathscr{F}^{\mathcal{I}}$-stopping times $\left.\mathcal{S}\right\}$ is uniformly integrable. This provides a second notion of stochastic intensity as follows.

Definition 2.5. By using Doob-Meyer's decomposition (see for instance Theorem I.3.15 of [18]), there is an increasing $\mathscr{F}^{\mathcal{I}, \tau}$-predictable process which is the compensator of $N$. We further assume that this compensator is absolutely continuous with respect to the Lebesgue measure with a non-negative Radon-Nikodym derivative $\lambda=\left(\lambda_{t}\right)_{t \geq 0}$ which is

\footnotetext{
${ }^{2}$ In [16], the filtration expansions (2) and (3) are respectively called minimal and progressive filtration expansions.
} 
an $\mathscr{F}^{\mathcal{I}}$-adapted càdlàg process. This implies that $\left(N_{t}-\int_{0}^{t \wedge \tau} \lambda_{u} d u\right)_{t \geq 0}$ is a uniformly integrable $\mathscr{F}^{\mathcal{I}, \tau}$-martingale. The process $\lambda$ is called the martingale intensity process (or sometimes just simply intensity) of $\tau$.

Note that in either one of the above definitions, a predictable version of intensity could be considered; for example since for each $t \geq 0, \int_{0}^{t \wedge \tau} \lambda_{u} d u=\int_{0}^{t \wedge \tau} \lambda_{u^{-}} d u$, instead of $\lambda$, one can use $\lambda_{-}$which is predictable.

An intuitive reason for the process $\lambda$ in Definition 2.5 to model the arrival rates of default is due to results such as Aven's theorem [2] that uses Meyer's Laplacian approximation. For a thorough discussion of the existence, calculation techniques, and examples of the intensity process in the sense of Definition 2.5, we refer to [16].

Remark 2.4. - If $\lambda$ is a hazard rate process in the sense of Definition 2.4 and if $\mathscr{F}^{\mathcal{I}, \tau}$ is the progressive filtration expansion $\mathscr{F}^{\mathcal{I}} \vee \mathcal{N}$ then by Proposition 5.1.3 of [6], $\left(\lambda_{t} 1_{\{t \leq \tau\}}\right)_{t \geq 0}$ is in fact an intensity process in the sense of Definition 2.5, but the converse of this statement does not necessarily hold. Therefore, by adopting the notion of intensity in the sense of Definition 2.5, we can also study models based on Definitions 2.4.

- Note that the existence of the intensity process of $\tau$ in either senses implies that $\tau$ is a totally inaccessible stopping time (see Definition 4.19 of [17]) which results in the unpredictability of the default time.

Remember that since we are taking the perspective of investors, the observable asset values are modeled by the process $Y$. In our set-up, the hedging process stops after the default time $\tau$ and so, we do not analyze what happens thereafter. Therefore, we concentrate on the stopped process $Y^{\tau}$. First, we introduce some notation.

- The optional projection of $\widetilde{N}=\left(1-N_{t}\right)_{t \geq 0}=\left(1_{\{\tau>t\}}\right)_{t \geq 0}$ over the filtration $\mathscr{F}^{\mathcal{I}}$ is denoted by $Z$.

- Note that $Z$ is an $\mathscr{F}^{\mathcal{I}}$-supermartingale and admits a canonical decomposition (so, it is a special semimartingale), see for instance Theorem 7, Chapter III of [25]. We let this decomposition be given by $Z=Z_{0}+M^{(z)}-A^{(z)}$ where $M^{(z)}, M_{0}^{(z)}=0$, and $A^{(z)}, A_{0}^{(z)}=0$, are respectively a uniformly integrable $\mathscr{F}^{\mathcal{I}}$-martingale and an increasing (hence of finite variation) $\mathscr{F}^{\mathcal{I}}$-predictable process. 
We already obtained the canonical decomposition of the process $Y, Y=X_{0}+M_{\alpha}+A_{\alpha}$ in $\mathscr{F}^{\mathcal{I}}$. Note that the canonical decomposition of a special semimartingale in a filtration, is not preserved in an expanded one. For instance, there is no guarantee that a local martingale (an example of a special semimartingale) remains a local martingale in an expanded filtration. One advantage of using $Y^{\tau}$ instead of $Y$ is that we are able to obtain its canonical decomposition in $\mathscr{F}^{\mathcal{I}, \tau}$. The following lemma points out this.

First, we remind that $[Y, Z]$ stands for the quadratic covariation process of $Y$ and $Z$. From the properties of quadratic covariation we have that $[Y, Z]=\left[M_{\alpha}, M^{(z)}\right]$. The predictable quadratic covariation of $M_{\alpha}$ and $M^{(z)}$ with respect to $\mathscr{F}^{\mathcal{I}}$ is denoted by $\left\langle M_{\alpha}, M^{(z)}\right\rangle^{\mathscr{F}^{\mathcal{I}}}$. The definitions and some basic properties of (predictable) quadratic covariation are reviewed in Definition 7.1 of the Appendix and the discussions following it.

Lemma 2.2. The process $Y^{\tau}$ is a special semimartingale in the expanded filtration $\mathscr{F}^{\mathcal{I}, \tau}$, and its canonical decomposition is given by

$$
Y^{\tau}=Y_{0}+A_{\alpha \cdot \wedge \tau}+\int_{0}^{\cdot \wedge \tau} \frac{1}{Z_{-}} d[Y, Z]+M^{(1)}
$$

where $A_{\alpha_{s}}=\int_{0}^{\alpha_{s}} a_{u} d u, s \geq 0$, and $M^{(1)}, M_{0}^{(1)}=0$, is an $\mathscr{F}^{\mathcal{I}, \tau}$-local martingale.

Proof. Note that $A_{\alpha_{\cdot \wedge \tau}}$ is $\mathscr{F}^{\mathcal{I}, \tau}$-predictable and of finite variation. Hence, the key step to find the canonical decomposition of $Y^{\tau}=X_{0}+M_{\alpha \cdot \wedge \tau}+A_{\alpha \cdot \wedge \tau}$ in $\mathscr{F}^{\mathcal{I}, \tau}$, is to obtain the canonical decomposition of $M_{\alpha \cdot \wedge \tau}$ in $\mathscr{F}^{\mathcal{I}, \tau}$.

Remember that $M^{(z)}$ is a uniformly integrable $\mathscr{F}^{\mathcal{I}}$-martingale, and hence it is the local martingale part of the canonical decomposition of $Z$ in $\mathscr{F}^{\mathcal{I}}$. Since $Z$ is uniformly bounded $^{3}$, it is easy to show that $M^{(z)}$ is a locally square integrable martingale. By Lemma 2.1, $M_{\alpha}$ is continuous, hence locally uniformly bounded and so locally square integrable. Note that $[Y, Z]=\left[M_{\alpha}, M^{(z)}\right]$ and since both $M_{\alpha}$ and $M^{(z)}$ are locally square integrable martingales, $\left\langle M_{\alpha}, M^{(z)}\right\rangle^{\mathscr{F}^{\mathcal{I}}}$ is well-defined. In fact, since $M_{\alpha}$ is continuous, the process $\left[M_{\alpha}, M^{(z)}\right]$ is also continuous and so we have $[Y, Z]=\left\langle M_{\alpha}, M^{(z)}\right\rangle^{\mathscr{F}^{\mathcal{I}}}$. Therefore, one can apply Theorem 7.1 of the Appendix for $\mathfrak{M}:=M_{\alpha}, \mathcal{F}:=\mathscr{F}^{\mathcal{I}}, Z^{\mathcal{F}}:=Z$, and $\mathcal{T}:=\tau$ which leads to the decomposition given by (5).

It only remains to show that (5) is in fact the canonical decomposition. The processes $[Y, Z], Z_{-}$, and $\alpha$ are $\mathscr{F}^{\mathcal{I}, \tau}$-predictable, and so Proposition I.3.5 of [18] shows that the

\footnotetext{
${ }^{3}$ The stochastic process $X$ is called uniformly bounded if there is a constant $c>0$ such that for all $t \geq 0$ and all $\omega \in \Omega$, we have $\left|X_{t}(\omega)\right| \leq c$, and it is called locally uniformly bounded, if it is locally in the class of uniformly bounded processes.
} 
processes $\int_{0}^{\cdot \wedge \tau} \frac{1}{Z_{-}} d[Y, Z]$ and $A_{\alpha_{\cdot \wedge \tau}}$ are $\mathscr{F}^{\mathcal{I}, \tau}$-predictable as well. Finally, Corollary I.3.16 of [18] implies that (5) is indeed the unique canonical decomposition of $Y^{\tau}$ in $\mathscr{F}^{\mathcal{I}, \tau}$.

Remark 2.5. In order to obtain an explicit form for the canonical decomposition of $Y^{\tau}$ in $\mathscr{F}^{\mathcal{I}, \tau}$, using Lemma 2.2, we need the explicit form of the canonical decomposition of $Z$ in $\mathscr{F}^{\mathcal{I}}$ (i.e. to determine $M^{(z)}$ ) which is not an easy problem by itself. Fortunately, for our main results in this paper, no explicit form of the canonical decomposition of $Z$, in $\mathscr{F}^{\mathcal{I}}$ is required, and this provides an advantage in applications.

\subsection{The Risk Management Methodology}

Finally, we need to determine the methodology that investors use to gauge and manage the risk of defaultable claims. We achieve this by finding PLRM (or the closely related one LRM) hedging strategies. This method has already been applied to hedge the risk of defaultable claims, see [4] and [5] for example. In this section, we present a short review of the PLRM approach and its connection to the LRM method within our framework; for details and discussions in a general set-up, we refer to [28].

Given a defaultable claim with payoff $H 1_{\{\tau>T\}}$, since we are taking the perspective of investors, what they actually observe is $Y=X_{\alpha}$ under the filtration $\mathscr{F}^{\mathcal{I}}$ where $H$ is $\mathscr{F}_{T}^{\mathcal{I}}$-measurable. However, as it is explained earlier, their proper filtration in which the hedging must take place is $\mathscr{F}^{\mathcal{I}, \tau}$ where $H 1_{\{\tau>T\}}$ is measurable.

Let $A^{(Y)}=A_{\alpha_{\cdot \wedge \tau}}+\int_{0}^{\cdot \wedge \tau} \frac{1}{Z_{-}} d[Y, Z]$ so that Equation (5) becomes $Y^{\tau}=Y_{0}+A^{(Y)}+M^{(1)}$.

Definition 2.6. Define $\Theta$ to be the space of all $\mathscr{F}^{\mathcal{I}, \tau}$-predictable processes $\theta$ such that $\mathbb{E}\left[\int_{0}^{T} \theta_{s}^{2} d\left[M^{(1)}\right]_{s}+\left(\int_{0}^{T}\left|\theta_{s} d A_{s}^{(Y)}\right|\right)^{2}\right]<\infty$.

Definition 2.7. An $L^{2}$-strategy is a pair $\phi=(\theta, \eta)$, where $\theta \in \Theta$ and $\eta$ is a real-valued $\mathscr{F}^{\mathcal{I}, \tau}$-adapted process such that the value process $V(\phi)=\theta Y^{\tau}+\eta$ is right-continuous and square-integrable, i.e. $V_{t}(\phi) \in L^{2}\left(\Omega, \mathscr{F}_{t}^{\mathcal{I}, \tau}, \mathbb{P}\right)$ for each $t \in[0, T]$.

In the above definition, $\theta$ and $\eta$ represent respectively the number of shares invested in the risky and the risk-free asset.

Following strategy $\phi$, the accumulated revenue (either profit or loss) from trading $Y^{\tau}$ on $[0, t], 0 \leq t \leq T$, is $\int_{0}^{t} \theta_{s} d Y_{s}^{\tau}$, and therefore the accumulated cost at time $0 \leq t \leq T$ from holding the portfolio based on $\phi$ is $C_{t}(\phi)=V_{t}(\phi)-\int_{0}^{t} \theta_{s} d Y_{s}^{\tau}$. A perfect strategy covers the claim completely, i.e. $V_{T}(\phi)=H 1_{\{\tau>T\}}$, P-a.s., and its cost is constant through time. However, as markets, in particular defaultable ones, are incomplete, this is not 
possible and so in return, according to the (local) risk-minimization approach, we look for those strategies that minimize the cost in some sense and lead to optimal strategies.

Suppose that an optimal hedging strategy is to cover a claim completely; though a constant cost process would be too much to ask for, it is reasonable to assume that the cost process of such optimal strategy should not deviate too much from its historical mean. This motivates the definition of a hedging strategy to be mean-self-financing if its cost process is an $\mathscr{F}^{\mathcal{I}, \tau}$-martingale.

Definition 2.8. An $L^{2}$-strategy $\phi=(\theta, \eta)$ is called mean-self-financing if its cost process $\left(C_{t}(\phi)\right)_{0 \leq t \leq T}$, defined by $C_{t}(\phi)=V_{t}(\phi)-\int_{0}^{t} \theta_{s} d Y_{s}^{\tau}, 0 \leq t \leq T$, is an $\mathscr{F}^{\mathcal{I}, \tau}$-martingale.

Definition 2.9. The risk process $R=\left(R_{t}(\phi)\right)_{0 \leq t \leq T}$, associated with the strategy $\phi$ and filtration $\mathscr{F}^{\mathcal{I}, \tau}$, is defined by $R_{t}(\phi)=E\left[\left(C_{T}(\phi)-C_{t}(\phi)\right)^{2} \mid \mathscr{F}_{t}^{\mathcal{I}, \tau}\right], 0 \leq t \leq T$.

The main idea in risk-minimization is to minimize the risk process $R$ in the following sense.

Definition 2.10. An $L^{2}$-strategy $\phi=(\theta, \eta)$ is called risk-minimizing if $V_{T}(\phi)=H 1_{\{\tau>T\}}$, $\mathbb{P}$-a.s., and for any $L^{2}$-strategy $\phi^{\prime}$ such that $V_{T}\left(\phi^{\prime}\right)=V_{T}(\phi)$, $\mathbb{P}$-a.s., we have $R_{t}(\phi) \leq$ $R_{t}\left(\phi^{\prime}\right), \mathbb{P}$-a.s., for every $0 \leq t \leq T$.

If $Y^{\tau}$ is an $\mathscr{F}^{\mathcal{I}, \tau}$-local martingale (i.e. $A^{(Y)}=0$ ) then the existence of such riskminimizing strategy (which is also mean-self-financing) is guaranteed. In this case the strategies are provided through Galtchouk-Kunita-Watanabe (GKW) decomposition of the claim which in our context states that the claim $H 1_{\{\tau>T\}}$ can be uniquely represented as $H 1_{\{\tau>T\}}=H_{0}+\int_{0}^{T} \theta_{s} d Y_{s}^{\tau}+L_{T}$, where $H_{0}=\mathbb{E}\left[H 1_{\{\tau>T\}} \mid \mathscr{F}_{0}^{\mathcal{I}, \tau}\right], \theta \in \Theta$, and $L$ is an $\mathscr{F}^{\mathcal{I}, \tau}$-square integrable martingale strongly orthogonal to $\int \theta^{\prime} d Y^{\tau}$ for all $\theta^{\prime} \in \Theta$, i.e. $L \int \theta^{\prime} d Y^{\tau}$ is an $\mathscr{F}^{\mathcal{I}, \tau}$-martingale.

For semimartingales a risk-minimizing optimal $L^{2}$-strategy in the above sense does not always exist, see [28] for a counterexample. In this case, risk minimization should be altered to LRM which is based on the idea that a strategy is optimal if its changes over a small interval of time does not cause an increase of risk. The exact definitions is rather technical and can be found in [28]. Instead of LRM, we focus on PLRM which is a closely related concept and easier to understand. The relationship between the two approaches will be explained shortly.

Definition 2.11. If we assume that $M^{(1)}$ is an $\mathscr{F}^{\mathcal{I}, \tau}$-square integrable martingale, an $L^{2}$-strategy $\phi$ with $V_{T}(\phi)=H 1_{\{\tau>T\}}, \mathbb{P}$-a.s., is called PLRM if it is mean-self-financing 
and its cost process $\left(C_{t}(\phi)\right)_{0 \leq t \leq T}$ is strongly orthogonal to $M^{(1)}$, i.e. $\left(C_{t}(\phi) M_{t}^{(1)}\right)_{0 \leq t \leq T}$ is an $\mathscr{F}^{\mathcal{I}, \tau}$-martingale.

The advantage of PLRM is that it is equivalent of finding a decomposition known as Föllmer-Schweizer (FS) decomposition. More precisely, by Proposition 3.4 of [28], the contingent claim $H 1_{\{\tau>T\}}$ admits a PLRM strategy $\phi$ if and only if $H 1_{\{\tau>T\}}$ can be written as

$$
H 1_{\{\tau>T\}}=H_{0}+\int_{0}^{T} \theta_{s} d Y_{s}^{\tau}+L_{T},
$$

where the equality holds $\mathbb{P}$-a.s., $H_{0} \in L^{2}\left(\Omega, \mathscr{F}_{0}^{\mathcal{I}, \tau}, \mathbb{P}\right)$ is the initial cost to start the hedging process, the process $\theta$ is $\mathscr{F}^{\mathcal{I}, \tau}$-predictable and belongs to $\Theta$, and $L, L_{0}=0$, is an $\mathscr{F}^{\mathcal{I}, \tau}$-square integrable martingale strongly orthogonal to $M^{(1)}$, i.e. $L M^{(1)}$ is an $\mathscr{F}^{\mathcal{I}, \tau_{-}}$ martingale.

Note that $L M^{(1)}$ is an $\mathscr{F}^{\mathcal{I}, \tau}$-martingale if and only if $\left\langle L, M^{(1)}\right\rangle^{\mathscr{F}^{\mathcal{I}, \tau}}=0$. This holds because if $L M^{(1)}$ is an $\mathscr{F}^{\mathcal{I}, \tau}$-martingale, by Lemma 7.1 of the Appendix, $\left\langle L, M^{(1)}\right\rangle^{\mathscr{F} \mathcal{I}, \tau}=$ 0 . On the other hand, if $\left\langle L, M^{(1)}\right\rangle^{\mathscr{F} \mathcal{I}, \tau}=0$, then by Theorem I.4.2 of [18], $L M^{(1)}$ is a uniformly integrable $\mathscr{F}^{\mathcal{I}, \tau}$-martingale.

In this case, the value process of the portfolio $\left(V_{t}(\phi)\right)_{t \geq 0}$ associated with the strategy $\phi$ is equal to

$$
V_{t}(\phi)=H_{0}+\int_{0}^{t} \theta_{s} d Y_{s}^{\tau}+L_{t}, \quad 0 \leq t \leq T
$$

the number of shares to be invested in the risky and risk-free asset are respectively equal to $\theta$ and $\eta_{t}=V_{t}(\phi)-\theta_{t} Y_{t}^{\tau}, 0 \leq t \leq T$, and finally the cost process is given by $C_{t}(\phi)=H_{0}+L_{t}, 0 \leq t \leq T$.

Equation (6) is known as the FS decomposition, and it was first introduced in [13]. We keep in mind that this decomposition leads to just PLRM hedging strategies and not necessarily to LRM strategies. In what follows, we discuss conditions under which the two methods are equivalent, i.e. they lead to the same hedging strategies.

More precisely, first one needs to check that the so called structure condition (SC) is satisfied, i.e. there exists an $\mathscr{F}^{\mathcal{I}, \tau}$-predictable process $\zeta$ such that $A^{(Y)}=\int \zeta d\left[M^{(1)}\right]$, and then to check the $\mathbb{P}$-a.s. finiteness of the process $\hat{K}$ (called the mean-variance trade-off process) defined by $\hat{K}=\int \zeta^{2} d\left[M^{(1)}\right]$. Since $Y$ is continuous and the model is supposed to be arbitrage-free, the SC condition is implied from Theorem 1 of [27].

So in our work, the SC condition is satisfied; if in addition, we also have that $M^{(1)}$ is an $\mathscr{F}^{\mathcal{I}, \tau}$-square integrable martingale, $\left\langle M^{(1)}\right\rangle^{\mathscr{F}^{\mathcal{I}, \tau}}$ is $\mathbb{P}$-a.s., strictly increasing, and $\mathbb{E}\left[\hat{K}_{T}\right]<\infty$, then by Theorem 3.3 of $[28]$, PLRM and LRM strategies are the same. 
Note that the equivalence between the two strategies (PLRM and LRM) holds in the one-dimensional case.

Therefore, the goal is to obtain the required FS decompositions and hence to determine the processes $\theta$ and $L$ in $\mathscr{F}^{\mathcal{I}, \tau}$ that satisfy (6). We address this challenge in the next section through canonical and KW decompositions (see Definition 7.3 of the Appendix) which lead to the FS decomposition of $H 1_{\{\tau>T\}}$ in $\mathscr{F}^{\mathcal{I}, \tau}$.

\section{$3 \quad$ KW and Canonical Decompositions}

The main purpose of this section is to apply and develop KW decomposition techniques that help finding FS decompositions and PLRM hedging strategies in Section 4. Note that the results of this section are still valid in the absence of any integrability assumptions on $Y$, and they hold under the existence of an intensity in the sense of Definition 2.5.

In what follows, in order to simplify the notation, when we write $\mathscr{F}^{\mathcal{I}, \tau} L M$ in an equation, we refer to an $\mathscr{F}^{\mathcal{I}, \tau}$-local martingale, and for $t \geq 0, \mathscr{F}^{\mathcal{I}, \tau} L M_{t}$ indicates the local martingale at $t$. Also, given a deterministic value $0<S \leq \infty$, the notation $h \in C^{1,2}([0, S) \times \mathbb{R})$ for a real-valued function $h$, means that $h:[0, S) \times \mathbb{R} \rightarrow \mathbb{R}$ is a $C^{1,2}$ function on $(0, S) \times \mathbb{R}$, and the indicated partial derivatives admit continuous extensions to $[0, S) \times \mathbb{R}$.

First, we obtain the canonical decomposition of the process $\left(h\left(\alpha_{t}, Y_{t}\right) \widetilde{N}_{t}\right)_{t \geq 0}$ in $\mathscr{F} \mathcal{I}, \tau$, where $\widetilde{N}=1-N=\left(1_{\{\tau>t\}}\right)_{t \geq 0}$ and $h \in C^{1,2}([0, \infty) \times \mathbb{R})$.

Proposition 3.1. Suppose that there exists an intensity $\lambda$ in the sense of Definition 2.5. Given a function $h \in C^{1,2}([0, \infty) \times \mathbb{R})$, the process $\left(h\left(\alpha_{t}, Y_{t}\right) \widetilde{N}_{t}\right)_{t \geq 0}$ is a special semimartingale in $\mathscr{F}^{\mathcal{I}, \tau}$, and its canonical decomposition is given by

$$
\begin{aligned}
h\left(\alpha_{t}, Y_{t}\right) \tilde{N}_{t}=h\left(0, Y_{0}\right) & -\int_{0}^{t \wedge \tau} h\left(\alpha_{s}, Y_{s}\right) \lambda_{s} d \gamma_{s}+\int_{0}^{t \wedge \tau} \mathcal{K}^{h}\left(\alpha_{s}, Y_{s}\right) d \alpha_{s} \\
& +\int_{0}^{t \wedge \tau} \frac{1}{Z_{s^{-}}} d[h(\alpha, Y), Z]_{s}+M_{t}^{(2)}, t \geq 0,
\end{aligned}
$$

where $\gamma_{t}=t-\alpha_{t}, t \geq 0, M^{(2)}, M_{0}^{(2)}=0$, is an $\mathscr{F}^{\mathcal{I}, \tau}$-local martingale, $Z$ is the optional projection of $\widetilde{N}$ over $\mathscr{F}^{\mathcal{I}}$, and for all $(t, x) \in[0, \infty) \times \mathbb{R}, \mathcal{K}^{h}(t, x)$ and $\mathcal{L}^{h}(t, x)$ are given by

$$
\begin{gathered}
\mathcal{K}^{h}(t, x)=\mathcal{L}^{h}(t, x)-h(t, x) \lambda_{t}, \\
\mathcal{L}^{h}(t, x)=\frac{\partial h}{\partial t}(t, x)+\frac{\partial h}{\partial x}(t, x) a_{t}+\frac{1}{2} \frac{\partial^{2} h}{\partial x^{2}}(t, x) b_{t},
\end{gathered}
$$


where the stochastic processes $\left(a_{t}\right)_{t \geq 0}$ and $\left(b_{t}\right)_{t \geq 0}$ are introduced in (1).

Proof. Using Itô's formula for the process $\left(h\left(\alpha_{t}, Y_{t}\right)\right)_{t \geq 0}$, we have

$$
h\left(\alpha_{t}, Y_{t}\right)=h\left(\alpha_{0}, Y_{0}\right)+\int_{0}^{t} \mathcal{L}^{h}\left(\alpha_{s}, Y_{s}\right) d \alpha_{s}+O_{t}, t \geq 0
$$

where $\mathcal{L}^{h}(t, x)=\frac{\partial h}{\partial t}(t, x)+\frac{\partial h}{\partial x}(t, x) a_{t}+\frac{1}{2} \frac{\partial^{2} h}{\partial x^{2}}(t, x) b_{t}$ and $O_{t}=\int_{0}^{t} \frac{\partial h}{\partial x}\left(\alpha_{s}, Y_{s}\right) d M_{\alpha_{s}}$. Note that $O=\left(O_{t}\right)_{t \geq 0}$ is an $\mathscr{F}^{\mathcal{I}}$-local martingale. Also, from the assumptions, the process

$$
\left(\tilde{N}_{t}+\int_{0}^{t \wedge \tau} \lambda_{s} d s\right)_{t \geq 0}=\left(1_{\{\tau>t\}}+\int_{0}^{t \wedge \tau} \lambda_{s} d \gamma_{s}+\int_{0}^{t \wedge \tau} \lambda_{s} d \alpha_{s}\right)_{t \geq 0}
$$

is an $\mathscr{F}^{\mathcal{I}, \tau}$-local martingale, because $\left(N_{t}-\int_{0}^{t \wedge \tau} \lambda_{s} d s\right)_{t>0}$ is a uniformly integrable $\mathscr{F}^{\mathcal{I}, \tau}$ martingale according to Definition 2.5. Therefore, for all $t \geq 0$, we have

$$
\widetilde{N}_{t}=-\int_{0}^{t \wedge \tau} \lambda_{s} d s+\mathscr{F}^{\mathcal{I}, \tau} L M_{t}=-\int_{0}^{t \wedge \tau} \lambda_{s} d \gamma_{s}-\int_{0}^{t \wedge \tau} \lambda_{s} d \alpha_{s}+\mathscr{F}^{\mathcal{I}, \tau} L M_{t} .
$$

On the other hand, since $O$ is continuous, it is a locally square integrable martingale, and so by Theorem 7.1 of the Appendix, the canonical decomposition of $O^{\tau}$ in $\mathscr{F}^{\mathcal{I}, \tau}$ is given by $O^{\tau}=O_{0}+\int_{0}^{\cdot \wedge \tau} \frac{1}{Z_{-}} d\left\langle O, M^{(z)}\right\rangle^{\mathscr{F}^{\mathcal{I}}}+\mathscr{F}^{\mathcal{I}, \tau} L M$, where $\left\langle O, M^{(z)}\right\rangle^{\mathscr{F}^{\mathcal{I}}}=$ $\langle O, Z\rangle^{\mathscr{F}^{\mathcal{I}}}=[h(\alpha, Y), Z]$. Therefore, by using the product rule, the canonical decomposition of $\left(h\left(\alpha_{t}, Y_{t}\right) \widetilde{N}_{t}\right)_{t \geq 0}$ is

$$
\begin{aligned}
h\left(\alpha_{t}, Y_{t}\right) 1_{\{\tau>t\}}= & h\left(0, Y_{0}\right)+\int_{0}^{t} h\left(\alpha_{s}, Y_{s}\right) d \widetilde{N}_{s}+\int_{0}^{t} 1_{\{s \leq \tau\}} d h\left(\alpha_{s}, Y_{s}\right) \\
= & h\left(0, Y_{0}\right)-\int_{0}^{t \wedge \tau} h\left(\alpha_{s}, Y_{s}\right) \lambda_{s} d \gamma_{s}-\int_{0}^{t \wedge \tau} h\left(\alpha_{s}, Y_{s}\right) \lambda_{s} d \alpha_{s}+\mathscr{F}^{\mathcal{I}, \tau} L M_{t} \\
& +\int_{0}^{t \wedge \tau}\left(\mathcal{L}^{h}\left(\alpha_{s}, Y_{s}\right)\right) d \alpha_{s}+\int_{0}^{t \wedge \tau} \frac{1}{Z_{s^{-}}} d[h(\alpha, Y), Z]_{s}+\mathscr{F}^{\mathcal{I}, \tau} L M_{t} \\
= & h\left(0, Y_{0}\right)-\int_{0}^{t \wedge \tau} h\left(\alpha_{s}, Y_{s}\right) \lambda_{s} d \gamma_{s} \\
& +\int_{0}^{t \wedge \tau} \mathcal{K}^{h}\left(\alpha_{s}, Y_{s}\right) d \alpha_{s}+\int_{0}^{t \wedge \tau} \frac{1}{Z_{s^{-}}} d[h(\alpha, Y), Z]_{s}+M_{t}^{(2)}, t \geq 0,
\end{aligned}
$$

where $M^{(2)}$ is an $\mathscr{F}^{\mathcal{I}, \tau}$-local martingale and $\mathcal{K}^{h}(t, x)=\mathcal{L}^{h}(t, x)-h(t, x) \lambda_{t}$, for all $(t, x) \in$ $[0, \infty) \times \mathbb{R}$. Since $\gamma, \alpha$, and $[h(\alpha, Y), Z]$ are $\mathscr{F}^{\mathcal{I}, \tau}$-predictable processes of finite variation, the integral terms are $\mathscr{F}^{\mathcal{I}, \tau}$-predictable and of finite variation, and this together with Corollary I.3.16 of [18] prove the uniqueness of the decomposition. 
In the next proposition, we obtain a closed-form formula for the $\mathrm{KW}$ decomposition of $M^{(2)}$ versus $M^{(1)}$ where $M^{(1)}$ and $M^{(2)}$ are defined respectively in Lemma 2.2 and Proposition 3.1.

Proposition 3.2. Suppose that there exists an intensity $\lambda$ in the sense of Definition 2.5. Given a function $h \in C^{1,2}([0, \infty) \times \mathbb{R})$, let $M^{(1)}$ and $M^{(2)}$ be respectively the $\mathscr{F}^{\mathcal{I}, \tau}$-local martingales in Lemma 2.2 and Proposition 3.1. Then $M^{(2)}$ admits a $K W$ decomposition versus $M^{(1)}$, in $\mathscr{F}^{\mathcal{I}, \tau}$, i.e. $M^{(2)}=\int \theta d M^{(1)}+L$, where $\theta=\frac{\partial h}{\partial x}(\alpha, Y) \tilde{N}_{-}$, and $L$ given by $\left(-h\left(\alpha_{\tau}, Y_{\tau}\right) N_{t}+\int_{0}^{t \wedge \tau} h\left(\alpha_{s}, Y_{s}\right) \lambda_{s} d s\right)_{t>0}$ is an $\mathscr{F}^{\mathcal{I}, \tau}$-local martingale orthogonal (see Definition 7.2 of the Appendix) to $M^{(1)}$.

Proof. From Proposition 3.1, we have

$$
\begin{aligned}
M^{(2)}=h(\alpha, Y) \tilde{N} & -h\left(0, Y_{0}\right)+\int_{0}^{\wedge \wedge \tau} h(\alpha, Y) \lambda d \gamma-\int_{0}^{\cdot \wedge \tau} \mathcal{K}^{h}(\alpha, Y) d \alpha \\
& -\int_{0}^{\wedge \wedge \tau} \frac{1}{Z_{-}} d[h(\alpha, Y), Z] .
\end{aligned}
$$

By Itô's formula, we get $h(\alpha, Y)=\int \frac{\partial h}{\partial t}(\alpha, Y) d \alpha+\int \frac{\partial h}{\partial x}(\alpha, Y) d Y+\frac{1}{2} \int \frac{\partial^{2} h}{\partial x^{2}}(\alpha, Y) d[Y]$. So, we have $[h(\alpha, Y), Z]=\int \frac{\partial h}{\partial x}(\alpha, Y) d[Y, Z]$ or in differential notation, $d[h(\alpha, Y), Z]=$ $\frac{\partial h}{\partial x}(\alpha, Y) d[Y, Z]$. Therefore, we obtain

$$
\begin{aligned}
M^{(2)}=h(\alpha, Y) \widetilde{N} & -h\left(0, Y_{0}\right)+\int_{0}^{\cdot \wedge \tau} h(\alpha, Y) \lambda d \gamma-\int_{0}^{\cdot \wedge \tau} \mathcal{K}^{h}(\alpha, Y) d \alpha \\
& -\int_{0}^{\wedge} \frac{\partial h}{\partial x}(\alpha, Y) \frac{1}{Z_{-}} d[Y, Z] .
\end{aligned}
$$

Note that by Proposition 3.1 for $(t, x) \in[0, \infty) \times \mathbb{R}, \mathcal{K}^{h}(t, x)=\mathcal{L}^{h}(t, x)-h(t, x) \lambda_{t}$, $\gamma_{t}=t-\alpha_{t}$, and from Lemma 2.2 , we have $\int_{0}^{\cdot \wedge \tau} \frac{1}{Z_{-}} d[Y, Z]=Y^{\tau}-Y_{0}-A_{\alpha_{\wedge} \wedge \tau}-M^{(1)}=$ $M_{\alpha \cdot \wedge \tau}-M^{(1)}$, substituting these equations in (7) leads to

$$
\begin{aligned}
M_{t}^{(2)}=h\left(\alpha_{t}, Y_{t}\right) \tilde{N}_{t} & -h\left(0, Y_{0}\right)+\int_{0}^{t \wedge \tau} h\left(\alpha_{s}, Y_{s}\right) \lambda_{s} d s-\int_{0}^{t \wedge \tau} \mathcal{L}^{h}\left(\alpha_{s}, Y_{s}\right) d \alpha_{s} \\
& -\int_{0}^{t \wedge \tau} \frac{\partial h}{\partial x}\left(\alpha_{s}, Y_{s}\right) d M_{\alpha_{s}}+\int_{0}^{t \wedge \tau} \frac{\partial h}{\partial x}\left(\alpha_{s}, Y_{s}\right) d M_{s}^{(1)}, t \geq 0 .
\end{aligned}
$$

By Itô's formula, we get $h\left(\alpha_{\cdot \wedge \tau}, Y_{\cdot \wedge \tau}\right)=h\left(0, Y_{0}\right)+\int_{0}^{\cdot \wedge \tau} \mathcal{L}^{h}(\alpha, Y) d \alpha+\int_{0}^{\cdot \wedge \tau} \frac{\partial h}{\partial x}(\alpha, Y) d M_{\alpha}$. Hence, for all $t \geq 0$, from (8), we obtain

$$
M_{t}^{(2)}=h\left(\alpha_{t}, Y_{t}\right) \tilde{N}_{t}-h\left(\alpha_{t \wedge \tau}, Y_{t \wedge \tau}\right)+\int_{0}^{t \wedge \tau} h\left(\alpha_{s}, Y_{s}\right) \lambda_{s} d s+\int_{0}^{t \wedge \tau} \frac{\partial h}{\partial x}\left(\alpha_{s}, Y_{s}\right) d M_{s}^{(1)}
$$


Finally by noting that for every $t \geq 0, h\left(\alpha_{t}, Y_{t}\right) \tilde{N}_{t}-h\left(\alpha_{t \wedge \tau}, Y_{t \wedge \tau}\right)=-h\left(\alpha_{\tau}, Y_{\tau}\right) N_{t}$, and by defining $L$ as the stochastic process $\left(-h\left(\alpha_{\tau}, Y_{\tau}\right) N_{t}+\int_{0}^{t \wedge \tau} h\left(\alpha_{s}, Y_{s}\right) \lambda_{s} d s\right)_{t \geq 0}$, we get $M^{(2)}=\int \theta d M^{(1)}+L$. Since $M^{(2)}$ and $\int \theta d M^{(1)}$ are $\mathscr{F}^{\mathcal{I}, \tau}$-local martingales, $L$ is also an $\mathscr{F}^{\mathcal{I}, \tau}$-local martingale. However, $L$ is obviously locally square integrable and of finite variation; therefore, $\left[L, M^{(1)}\right]=0$ which means that $\left\langle L, M^{(1)}\right\rangle^{\mathscr{F} \mathcal{I}, \tau}=0$. Then by Lemma 7.1 of the Appendix, $L$ is orthogonal to $M^{(1)}$.

Remark 3.1. Note that although $\tau$ is an $\mathscr{F}^{\mathcal{I}, \tau}$-totally inaccessible stopping time (loosely speaking, this means that it is completely unpredictable), the process $\theta=\frac{\partial h}{\partial x}(\alpha, Y) \widetilde{N}_{-}=$ $\frac{\partial h}{\partial x}(\alpha, Y) 1_{[0, \tau]}$ in Proposition 3.2 is $\mathscr{F}^{\mathcal{I}, \tau}$-predictable. This is because $Y$, $\alpha$, and $\widetilde{N}_{-}$are all predictable in $\mathscr{F}^{\mathcal{I}, \tau}$.

The next theorem is vital in obtaining FS decompositions in the next section. Recall that $Y=X_{\alpha}$ and $\alpha$ are respectively the investors' asset observation and the random time change to model the delay as in Definition 2.1.

Theorem 3.1. Suppose that there exists an intensity $\lambda$ in the sense of Definition 2.5. Consider a function $h \in C^{1,2}([0, \infty) \times \mathbb{R})$ and an $\mathscr{F}^{\mathcal{I}, \tau}$-stopping time $\mathfrak{T}: \Omega \rightarrow[0, \infty)$.

1. If $\mathbb{P}$-a.s. on $\{\tau \geq t\}$ and $\{0 \leq t<\mathfrak{T}\}$, the following equation is satisfied d $\alpha$-a.s.,

$$
\frac{\partial h}{\partial t}\left(\alpha_{t}, Y_{t}\right)+\frac{1}{2} \frac{\partial^{2} h}{\partial x^{2}}\left(\alpha_{t}, Y_{t}\right) b_{\alpha_{t}}-\lambda_{t} h\left(\alpha_{t}, Y_{t}\right)=0
$$

then we have

$$
h\left(\alpha \cdot \wedge \mathfrak{T}, Y_{\cdot \wedge \mathfrak{T})} \tilde{N}_{\cdot \wedge \mathfrak{T}}+\int_{0}^{\cdot \wedge \mathfrak{T} \wedge \tau} h(\alpha, Y) \lambda d \gamma=h\left(0, X_{0}\right)+\int_{0}^{\cdot \wedge \mathfrak{T}} \theta d Y^{\tau}+L_{\cdot \wedge \mathfrak{T}},\right.
$$

where for $t \geq 0, \gamma_{t}=t-\alpha_{t}, \theta_{t}=\frac{\partial h}{\partial x}\left(\alpha_{t}, Y_{t}\right) \tilde{N}_{t^{-}}, \tilde{N}_{t}=1-N_{t}=1_{\{\tau>t\}}, L_{t}=$ $-h\left(\alpha_{\tau}, Y_{\tau}\right) N_{t}+\int_{0}^{t \wedge \tau} h\left(\alpha_{s}, Y_{s}\right) \lambda_{s} d s$, and $L_{. \wedge \mathfrak{T}}=\left(L_{t \wedge \mathfrak{T}}\right)_{t \geq 0}$, is an $\mathscr{F}^{\mathcal{I}, \tau}$-local martingale orthogonal to both $M^{(1)}$ and $M_{\cdot \wedge \mathfrak{T}}^{(1)}\left(M^{(1)}\right.$ is the local martingale part of $Y^{\tau}$ as in Lemma 2.2). Furthermore, the equality holds up to an evanescent set.

2. If $\alpha$ is $\mathbb{P}$-almost surely absolutely continuous with respect to the Lebesgue measure, with the Radon-Nikodym derivative $\frac{d \alpha_{t}}{d t}$, and if $\mathbb{P}$-a.s. on $\{\tau \geq t\}$ and $\{0 \leq t<\mathfrak{T}\}$, the following equation is satisfied Lebesgue-a.s.,

$$
\frac{d \alpha_{t}}{d t}\left(\frac{\partial h}{\partial t}\left(\alpha_{t}, Y_{t}\right)+\frac{1}{2} \frac{\partial^{2} h}{\partial x^{2}}\left(\alpha_{t}, Y_{t}\right) b_{\alpha_{t}}\right)-\lambda_{t} h\left(\alpha_{t}, Y_{t}\right)=0
$$


then we have

$$
h\left(\alpha_{\cdot \wedge \mathfrak{T}}, Y_{\cdot \wedge \mathfrak{T}}\right) \widetilde{N}_{\cdot \wedge \mathfrak{T}}=h\left(0, X_{0}\right)+\int_{0}^{\cdot \wedge \mathfrak{T}} \theta d Y^{\tau}+L_{\cdot \wedge \mathfrak{T}},
$$

where for $t \geq 0, \theta_{t}=\frac{\partial h}{\partial x}\left(\alpha_{t}, Y_{t}\right) \widetilde{N}_{t^{-}}, L_{t}=-h\left(\alpha_{\tau}, Y_{\tau}\right) N_{t}+\int_{0}^{t \wedge \tau} h\left(\alpha_{s}, Y_{s}\right) \lambda_{s} d s$, and $L \cdot \wedge \mathfrak{T}=\left(L_{t \wedge \mathfrak{T}}\right)_{t \geq 0}$ is an $\mathscr{F}^{\mathcal{I}, \tau}$-local martingale orthogonal to both $M^{(1)}$ and $M_{\cdot \wedge \mathfrak{T}}^{(1)}$. Furthermore, the equality holds up to an evanescent set.

Proof. Recall that $M^{(2)}$ is the $\mathscr{F}^{\mathcal{I}, \tau}$-local martingale in Proposition 3.1. Because $\mathfrak{T}$ is an $\mathscr{F}^{\mathcal{I}, \tau}$-stopping time, both $M_{\cdot \wedge \mathfrak{T}}^{(2)}$ and $M_{\cdot \wedge \mathfrak{T}}^{(1)}$ are $\mathscr{F}^{\mathcal{I}, \tau}$-local martingales, and from Proposition 3.2 , the KW decomposition of $M_{\cdot \wedge \mathfrak{T}}^{(2)} \operatorname{versus} M_{\cdot \wedge \mathfrak{T}}^{(1)}$ is equal to $M_{\cdot \wedge \mathfrak{T}}^{(2)}=\int \theta d M_{\cdot \wedge \mathfrak{T}}^{(1)}+L_{\cdot \wedge \mathfrak{T}}$, where $\theta=\frac{\partial h}{\partial x}(\alpha, Y) \tilde{N}_{-}$and $L$ is the $\mathscr{F}^{\mathcal{I}, \tau}$-local martingale of Proposition 3.2 which is

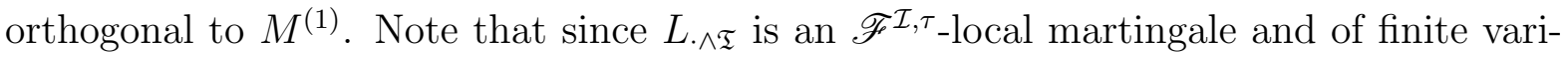
ation, $\left\langle L_{\cdot \wedge \mathfrak{T}}, M_{\cdot \wedge \mathfrak{T}}^{(1)}\right\rangle^{\mathscr{F} \mathcal{I}, \tau}=\left\langle L_{\cdot \wedge \mathfrak{T}}, M^{(1)}\right\rangle^{\mathscr{F} \mathcal{I}, \tau}=0$, and by Lemma 7.1 of the Appendix $L_{\cdot \wedge \mathfrak{T}}$ is orthogonal to both $M^{(1)}$ and $M_{\cdot \wedge \mathfrak{T}}^{(1)}$.

After plugging the formulas for $M^{(1)}$ (obtained from (5)) and $M^{(2)}$ (given by (7)) into the aforementioned KW decomposition of $M_{\cdot \wedge \mathfrak{T}}^{(2)}$ versus $M_{\cdot \wedge \mathfrak{T}}^{(1)}$, for $t \geq 0$, we get

$$
\begin{aligned}
h\left(\alpha_{t \wedge \mathfrak{T}}, Y_{t \wedge \mathfrak{T}}\right) \widetilde{N}_{t \wedge \mathfrak{T}} & -h\left(0, X_{0}\right)+\int_{0}^{t \wedge \mathfrak{T} \wedge \tau} h\left(\alpha_{s}, Y_{s}\right) \lambda_{s} d \gamma_{s}-\int_{0}^{t \wedge \mathfrak{T} \wedge \tau} \mathcal{K}^{h}\left(\alpha_{s}, Y_{s}\right) d \alpha_{s} \\
& =\int_{0}^{t \wedge \mathfrak{T}} \theta_{s} d Y_{s}^{\tau}-\int_{0}^{t \wedge \mathfrak{T} \wedge \tau} \frac{\partial h}{\partial x}\left(\alpha_{s}, Y_{s}\right) a_{\alpha_{s}} d \alpha_{s}+L_{t \wedge \mathfrak{T}}, \mathbb{P} \text {-a.s.. }
\end{aligned}
$$

Since $\mathbb{P}$-a.s. on $\{\tau \geq s\}$ and $0 \leq s<\mathfrak{T}$, condition (9) holds $d \alpha$-a.s., we have $\mathbb{P}$-a.s., $\mathcal{K}^{h}\left(\alpha_{s}, Y_{s}\right)=\frac{\partial h}{\partial x}\left(\alpha_{s}, Y_{s}\right) a_{\alpha_{s}}, d \alpha$-a.s., for all $0 \leq s<\mathfrak{T}$. In addition, the measure $d \alpha$ does not charge $\{s \geq 0 ; \mathfrak{T}=s\}$, and so for all $t \geq 0$, we obtain

$$
h\left(\alpha_{t \wedge \mathfrak{T}}, Y_{t \wedge \mathfrak{T}}\right) \tilde{N}_{t \wedge \mathfrak{T}}+\int_{0}^{t \wedge \mathfrak{T} \wedge \tau} h\left(\alpha_{s}, Y_{s}\right) \lambda_{s} d \gamma_{s}=h\left(0, X_{0}\right)+\int_{0}^{t \wedge \mathfrak{T}} \theta_{s} d Y_{s}^{\tau}+L_{t \wedge \mathfrak{T}}, \mathbb{P} \text {-a.s.. }
$$

The left and right-hand sides of the previous equation define two processes which are modifications of each other. Since they are càdlàg processes, they are equal up to an evanescence set. This proves part 1.

Now we show part 2 of the theorem. Since $\alpha$ is absolutely continuous with respect to the Lebesgue measure, for $0 \leq t \leq \mathfrak{T}$, (11) becomes

$$
\begin{aligned}
h\left(\alpha_{t}, Y_{t}\right) \tilde{N}_{t} & -h\left(0, X_{0}\right)+\int_{0}^{t \wedge \tau} h\left(\alpha_{s}, Y_{s}\right) \lambda_{s} d s-\int_{0}^{t \wedge \tau}\left(\mathcal{K}^{h}\left(\alpha_{s}, Y_{s}\right)+h\left(\alpha_{s}, Y_{s}\right) \lambda_{s}\right) \frac{d \alpha_{s}}{d s} d s \\
& =\int_{0}^{t} \theta_{s} d Y_{s}^{\tau}-\int_{0}^{t \wedge \tau} \frac{\partial h}{\partial x}\left(\alpha_{s}, Y_{s}\right) a_{\alpha_{s}} \frac{d \alpha_{s}}{d s} d s+L_{t}, \mathbb{P} \text {-a.s.. }
\end{aligned}
$$


Part 2 can be then obtained in a similar way, by noting that $\mathbb{P}$-a.s. on $\{\tau \geq s\}, 0 \leq s<\mathfrak{T}$, we have $h\left(\alpha_{s}, Y_{s}\right) \lambda_{s}-\left(\mathcal{K}^{h}\left(\alpha_{s}, Y_{s}\right)+h\left(\alpha_{s}, Y_{s}\right) \lambda_{s}\right) \frac{d \alpha_{s}}{d s}=-\frac{\partial h}{\partial x}\left(\alpha_{s}, Y_{s}\right) a_{\alpha_{s}} \frac{d \alpha_{s}}{d s}$, Lebesgue-a.s..

Remark 3.2. The results of Theorem 3.1 are still valid if $\mathfrak{T}=\infty$. In fact, the same lines of proof would work.

The next lemma considers the case of no delay in data. This puts both investors and managers in the same position with respect to the underlying asset.

Lemma 3.1. Under the same assumption as Theorem 3.1, suppose that there is no delay in receiving the data, i.e. $\alpha_{t}=t$ for all $t \geq 0$. If $\mathbb{P}$-a.s. on $\{\tau \geq t\}$ and $\{0 \leq t<\mathfrak{T}\}$, the following equation is satisfied Lebesgue-a.s., for a function $h \in C^{1,2}([0, \infty) \times \mathbb{R})$,

$$
\frac{\partial h}{\partial t}\left(t, X_{t}\right)+\frac{1}{2} \frac{\partial^{2} h}{\partial x^{2}}\left(t, X_{t}\right) b_{t}-\lambda_{t} h\left(t, X_{t}\right)=0
$$

then we have

$$
h\left(\cdot \wedge \mathfrak{T}, X_{\cdot \wedge \mathfrak{T})}\right) \tilde{N}_{\cdot \wedge \mathfrak{T}}=h\left(0, X_{0}\right)+\int_{0}^{\cdot \wedge \mathfrak{T}} \theta d X^{\tau}+L_{\cdot \wedge \mathfrak{T}},
$$

where for $t \geq 0, \theta_{t}=\frac{\partial h}{\partial x}\left(t, X_{t}\right) \widetilde{N}_{t^{-}}, L_{t}=-h\left(\tau, X_{\tau}\right) N_{t}+\int_{0}^{t \wedge \tau} h\left(s, X_{s}\right) \lambda_{s} d s$, and $L \cdot \wedge \mathfrak{T}=$ $\left(L_{t \wedge \mathfrak{T}}\right)_{t \geq 0}$ is an $\mathscr{F}^{\mathcal{I}, \tau}$-local martingale orthogonal to the local martingale part of $X$ in $\mathscr{F}^{\mathcal{I}, \tau}$. Furthermore, the equality holds up to an evanescent set.

Proof. Since for all $t \geq 0, \alpha_{t}=t$, then $\gamma=0, Y=X$, and the result is a direct application of part 1 of Theorem 3.1 .

Remark 3.3. Note that for the $\mathscr{F}^{\mathcal{I}}$-semimartingale $Y$ and for all $t \geq 0$, we have $\int_{0}^{t} \theta_{s} d Y_{s}^{\tau}=\int_{0}^{t} \theta_{s} 1_{\{s \leq \tau\}} d Y_{s}^{\tau}=\int_{0}^{t} \theta_{s} 1_{\{s \leq \tau\}} d Y_{s}$, so in view of Theorem 3.1 and Lemma 3.1, if hedging is carried out according to the stopped process $Y^{\tau}$, we may assume that $\theta$ vanishes after $\tau$.

\section{Applications and Examples}

In this section, we present some results and examples in which FS decompositions and PLRM hedging strategies are obtained through semi-explicit solutions, based on PDEs.

Proposition 4.1. Consider a defaultable claim $(H, \tau, T)$ and suppose that there exists an intensity $\lambda$ in the sense of Definition 2.5. Assume that $\alpha:[0, \infty) \rightarrow[0, \infty)$ is a deterministic time change which is absolutely continuous with respect to the Lebesgue 
measure, and its Radon-Nikodym derivative with respect to the Lebesgue measure is both positive and continuous. Let $H=F\left(Y_{T}\right)$, and for $t \geq 0, \lambda_{t}=g\left(\alpha_{t}, Y_{t}\right), b_{t}=f\left(t, X_{t}\right)$, where $F: \mathbb{R} \rightarrow \mathbb{R}, g:[0, \infty) \times \mathbb{R} \rightarrow[0, \infty)$, and $f:[0, \infty) \times \mathbb{R} \rightarrow[0, \infty)$ are continuous functions. Suppose that there is a continuous function $h:[0, T] \times \mathbb{R} \rightarrow \mathbb{R}$, such that $h \in C^{1,2}([0, T) \times \mathbb{R})$, and $h$ is a solution of the following PDE:

$$
\begin{aligned}
& \frac{\partial h}{\partial t}(t, x)+\frac{1}{2} f\left(\alpha_{t}, x\right) \frac{d \alpha_{t}}{d t} \frac{\partial^{2} h}{\partial x^{2}}(t, x)-g\left(\alpha_{t}, x\right) h(t, x)=0,(t, x) \in[0, T) \times \mathbb{R}, \\
& h(T, x)=F(x) .
\end{aligned}
$$

Then we have

$$
H \widetilde{N}_{T}=h\left(0, X_{0}\right)+\int_{0}^{T} \theta_{s} 1_{\{s<T\}} d Y_{s}^{\tau}+L_{T},
$$

where $\theta_{t}=\frac{\partial h}{\partial x}\left(t, Y_{t}\right) 1_{\{t \leq \tau\}}, 0 \leq t<T$, and $L=\left(L_{t}\right)_{t \geq 0}, L_{0}=0$, is an $\mathscr{F}^{\mathcal{I}, \tau}$-local martingale orthogonal to $M^{(1)}$ (the local martingale part of $Y^{\tau}$ in $\mathscr{F}^{\mathcal{I}, \tau}$ as in Lemma 2.2).

Furthermore, suppose that $M^{(1)}$ and $L$ are square integrable martingales, and $\theta 1_{\{\cdot<T\}}$ belongs to $\Theta$ (see Definition 2.6). Then the FS decomposition of the defaultable claim is given by (13).

Proof. For all $(t, x) \in\left[0, \alpha_{T}\right] \times \mathbb{R}$, define $v(t, x)$ by $v(t, x)=h\left(\alpha_{t}^{-1}, x\right)$. Since $t \mapsto \frac{d \alpha_{t}}{d t}$ is positive and continuous, $v:\left[0, \alpha_{T}\right] \times \mathbb{R} \rightarrow \mathbb{R}$ is well-defined and $v \in C^{1,2}\left(\left[0, \alpha_{T}\right) \times \mathbb{R}\right)$. Furthermore, we have $h(t, x)=v\left(\alpha_{t}, x\right)$, for all $(t, x) \in[0, T] \times \mathbb{R}$, therefore (12) becomes

$$
\frac{d \alpha_{t}}{d t}\left(\frac{\partial v}{\partial t}\left(\alpha_{t}, x\right)+\frac{1}{2} f\left(\alpha_{t}, x\right) \frac{\partial^{2} v}{\partial x^{2}}\left(\alpha_{t}, x\right)\right)-g\left(\alpha_{t}, x\right) v\left(\alpha_{t}, x\right)=0,(t, x) \in[0, T) \times \mathbb{R} .
$$

This implies that

$$
\frac{d \alpha_{t}}{d t}\left(\frac{\partial v}{\partial t}\left(\alpha_{t}, Y_{t}\right)+\frac{1}{2} \frac{\partial^{2} v}{\partial x^{2}}\left(\alpha_{t}, Y_{t}\right) b_{\alpha_{t}}\right)-\lambda_{t} v\left(\alpha_{t}, Y_{t}\right)=0,0 \leq t<T
$$

At this stage, it might be tempting to use part 2 of Theorem 3.1 for $v$ straight away; however, in order to do so, $v$ needs to be $C^{1,2}$ on $[0, \infty) \times \mathbb{R}$. We fix this problem as follows.

For an integer $n \geq 1$, consider the restriction of $v$ on $\left[0, \alpha_{T-\frac{1}{n}}\right] \times \mathbb{R}$, denoted by

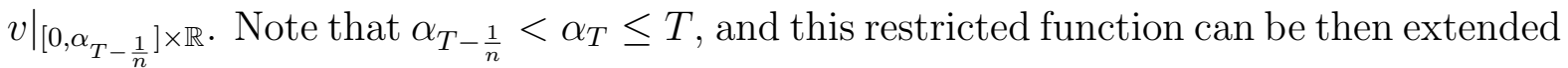
to a $C^{1,2}$ function on $[0, \infty) \times \mathbb{R}$ ) which we denote by $v^{(n)}$, for instance by Theorem I of [29]. Therefore, $v^{(n)} \in C^{1,2}([0, \infty) \times \mathbb{R})$ and $\left.v^{(n)}\right|_{\left[0, \alpha_{T-\frac{1}{n}}\right] \times \mathbb{R}}=v$; the latter indicates that for all $0 \leq t \leq T-\frac{1}{n}$ and $x \in \mathbb{R}, v^{(n)}\left(\alpha_{t}, x\right)=v\left(\alpha_{t}, x\right)^{n}=h(t, x)$. 
Let $S(n)=T-\frac{1}{n}, n \geq 1$, then part 2 of Theorem 3.1 can be applied to $v^{(n)}$ and $\mathfrak{T}=S(n)$ which leads to

$$
v^{(n)}\left(\alpha_{\cdot \wedge S(n)}, Y_{\cdot \wedge S(n)}\right) \tilde{N}_{\cdot \wedge S(n)}=v^{(n)}\left(0, X_{0}\right)+\int_{0}^{\cdot \wedge S(n)} \theta^{(n)} d Y^{\tau}+L_{\cdot \wedge S(n)}^{(n)},
$$

where for all $0 \leq t \leq T-\frac{1}{n}, \theta_{t}^{(n)}=\frac{\partial v^{(n)}}{\partial x}\left(\alpha_{t}, Y_{t}\right) 1_{\{t \leq \tau\}}$ which is equal to $\theta_{t}=\frac{\partial h}{\partial x}\left(t, Y_{t}\right) 1_{\{t \leq \tau\}}$, $L_{t}^{(n)}=-v^{(n)}\left(\alpha_{\tau}, Y_{\tau}\right) N_{t}+\int_{0}^{t \wedge \tau} v^{(n)}\left(\alpha_{s}, Y_{s}\right) \lambda_{s} d s, t \geq 0$, and $L_{\cdot \wedge S(n)}^{(n)}$ is orthogonal to $M_{\cdot \wedge S(n)}^{(1)}$. Next, we take the limit of (14) as $n \rightarrow \infty$.

Since $h$ is continuous and the distribution of $\tau$ has no atoms, by the construction of $v^{(n)}, v^{(n)}\left(\alpha_{\cdot \wedge S(n)}, Y_{\cdot \wedge S(n)}\right) \tilde{N}_{\cdot \wedge S(n)}$ converges to the process $v\left(\alpha_{\cdot \wedge T}, Y_{\cdot \wedge T}\right) \tilde{N}_{\cdot \wedge T}$, uniformly on compacts $^{4}, \mathbb{P}$-a.s., as $n \rightarrow \infty$. On the other hand, for all $t \geq 0$, we have that

$$
\int_{0}^{t \wedge S(n)} \theta_{s}^{(n)} d Y_{s}^{\tau}=\int_{0}^{t \wedge S(n)} \theta_{s} 1_{\{s<T\}} d Y_{s}^{\tau}
$$

Since $\theta 1_{\{\cdot<T\}}$ is $\mathscr{F}^{\mathcal{I}, \tau}$-predictable and locally bounded, the stochastic integral $\int \theta 1_{\{\cdot<T\}} d Y^{\tau}$ is well-defined. Obviously, $\int_{0}^{t \wedge S(n)} \theta_{s}^{(n)} d Y_{s}^{\tau}=\int_{0}^{t} \theta_{s} 1_{\{s<T\}} 1_{\left\{s \leq T-\frac{1}{n}\right\}} d Y_{s}^{\tau}, t \geq 0$, hence, by part (iii) of Theorem I.4.31 of [18], we have $\int_{0}^{\wedge \wedge(n)} \theta^{(n)} d Y^{\tau}$ converges to $\int_{0}^{\wedge \wedge T} \theta 1_{\{\cdot<T\}} d Y^{\tau}$, uniformly on compacts, in probability, as $n \rightarrow \infty$.

Also, $L_{t \wedge S(n)}^{(n)}=-v^{(n)}\left(\alpha_{\tau}, Y_{\tau}\right) N_{t \wedge S(n)}+\int_{0}^{t \wedge S(n) \wedge \tau} v^{(n)}\left(\alpha_{s}, Y_{s}\right) \lambda_{s} d s, t \geq 0$, which is equal to $L_{t \wedge S(n)}^{v}, t \geq 0$, where $L_{t}^{v}=-v\left(\alpha_{\tau}, Y_{\tau}\right) N_{t}+\int_{0}^{t \wedge \tau} v\left(\alpha_{s}, Y_{s}\right) \lambda_{s} d s, 0 \leq t \leq T$. It is easy to check that the sequence $\left\{L_{\cdot \wedge S(n)}^{(n)} ; n \geq 1\right\}$ converges to $\left(L_{t \wedge T}^{v}\right)_{t \geq 0}$, uniformly on compacts, $\mathbb{P}$-a.s., as $n \rightarrow \infty$. Let $L=\left(L_{t \wedge T}^{v}\right)_{t \geq 0}$, then from the definition of $L^{v}$, we can see that $L=L_{\cdot \wedge T}^{v}=-\int_{0}^{\cdot \wedge T \wedge \tau} v(\alpha, Y) d M^{\lambda}$, where $M^{\lambda}$, given by $M_{t}^{\lambda}=N_{t}-\int_{0}^{t \wedge \tau} \lambda_{s} d s, t \geq 0$, is a uniformly integrable $\mathscr{F}^{\mathcal{I}, \tau}$-martingale. Therefore, $L$ is an $\mathscr{F}^{\mathcal{I}, \tau}$-local martingale. Since $L$ is of finite variation, $\left\langle L, M^{(1)}\right\rangle^{\mathscr{F} \mathcal{I}, \tau}=0$, and so by Lemma 7.3 of the Appendix, it is orthogonal to $M^{(1)}$.

From the previous argument, the limit of all the terms in (14) exists uniformly on compacts in probability as $n \rightarrow \infty$, and so we obtain $v\left(\alpha_{\cdot \wedge T}, Y_{\cdot \wedge T}\right) \tilde{N}_{\cdot \wedge T}=v\left(0, X_{0}\right)+$ $\int_{0}^{\cdot \wedge T} \theta 1_{\{\cdot<T\}} d Y^{\tau}+L$, which leads to

$$
v\left(\alpha_{T}, Y_{T}\right) \widetilde{N}_{T}=v\left(0, X_{0}\right)+\int_{0}^{T} \theta_{s} 1_{\{s<T\}} d Y_{s}^{\tau}+L_{T}
$$

\footnotetext{
${ }^{4} \mathrm{~A}$ sequence of processes $\left\{X^{(n)} ; n \geq 1\right\}$ converges to a process $X^{*}$, uniformly on compacts, $\mathbb{P}$-a.s., as $n \rightarrow \infty$, if for each $t \geq 0$, $\sup _{0 \leq s \leq t}\left(\left|X_{s}^{(n)}-X_{s}^{*}\right|\right)$ converges to zero $\mathbb{P}$-a.s., as $n \rightarrow \infty$. Similarly, one can define uniformly on compacts convergence in probability, and it is obvious that the former implies the latter.
} 
Obviously $v\left(0, X_{0}\right)=h\left(0, X_{0}\right)$, and also from the boundary condition $h(T, x)=F(x)$, we have that $v\left(\alpha_{T}, x\right)=h(T, x)=F(x)$ or $v\left(\alpha_{T}, Y_{T}\right)=F\left(Y_{T}\right)=H$ which concludes the result.

Finally, since $\theta 1_{\{\cdot<T\}}$ belongs to $\Theta$, we have that (13) is the FS decomposition of $H \widetilde{N}_{T}$ in $\mathscr{F}^{\mathcal{I}, \tau}$.

Remark 4.1. Regarding Proposition 4.1, we make the following remarks:

- The indicator process $1_{\{\cdot<T\}}$ in $\int \theta 1_{\{\cdot<T\}} d Y^{\tau}$ is to ensure that $\theta 1_{\{\cdot<T\}}$ and hence $\int \theta 1_{\{\cdot<T\}} d Y^{\tau}$, are well defined. If $\frac{\partial h}{\partial x}(t, x)$ exists for $t=T$ and $x \in \mathbb{R}$, then $\theta_{T}$ is well-defined, and we have $\int_{0}^{T} \theta_{s} 1_{\{s<T\}} d Y_{s}^{\tau}=\int_{0}^{T} \theta_{s} d Y_{s}^{\tau}, \mathbb{P}$-a.s.. This means that whether or not $\theta$ is defined at $t=T$, it does not affect the FS decomposition.

- Under some assumptions, the solution of PDE (12) with its boundary condition can be found through Feynman-Kac formula (see for instance Chapter 5 of [7] or Section 4.4 of [22]) and numerical techniques.

- Note that the previous proposition does not cover the interesting cases where the time change is absolutely continuous with respect to the Lebesgue measure, but the Radon Nikodym derivative is not continuous, for instance $\alpha_{t}=(t-l)^{+}$, where $t \geq 0$ and $l>0$ is a constant. Nevertheless this case is discussed in Example 4.2.

A special case of the previous proposition is the case of no delay as follows.

Lemma 4.1. Keep the same assumption as in Proposition 4.1, and suppose that $\alpha_{t}=t$, for all $t \geq 0$. Furthermore, assume that there is a continuous function $h:[0, T] \times \mathbb{R} \rightarrow \mathbb{R}$, such that $h \in C^{1,2}([0, T) \times \mathbb{R})$, and $h$ is a solution of the following PDE:

$$
\begin{aligned}
& \frac{\partial h}{\partial t}(t, x)+\frac{1}{2} f(t, x) \frac{\partial^{2} h}{\partial x^{2}}(t, x)-g(t, x) h(t, x)=0,(t, x) \in[0, T) \times \mathbb{R}, \\
& h(T, x)=F(x) .
\end{aligned}
$$

Then we have

$$
H \widetilde{N}_{T}=h\left(0, X_{0}\right)+\int_{0}^{T} \theta_{s} 1_{\{s<T\}} d X_{s}^{\tau}+L_{T},
$$

where $L=\left(L_{t}\right)_{t \geq 0}, L_{0}=0$, is an $\mathscr{F}^{\mathcal{I}, \tau}$-local martingale orthogonal to the local martingale part of $X^{\tau}$ in $\mathscr{F}^{\mathcal{I}, \tau}$. Furthermore suppose that $L$ and the local martingale part of $X^{\tau}$ in $\mathscr{F}^{\mathcal{I}, \tau}$ are square integrable martingales, and $\theta 1_{\{\cdot<T\}}$ belongs to $\Theta$. Then the FS decomposition of the defaultable claim is given by (15). 
Proof. This is a direct application of Proposition 4.1 by letting $\alpha_{t}=t, t \geq 0$.

We continue by a very simple case in which no information is released from the firm to investors.

Example 4.1. Suppose that we would like to obtain the PLRM hedging strategy for a defaultable claim whose maturity, denoted by $T$, is before any information released from the firm. So we assume that there is a constant $l>0$ such that $T \leq l$ and for $0 \leq t \leq l$ no information is released from the firm which means that the investors' information is not updated, i.e. $\alpha_{t}=0$ for all $0 \leq t \leq l$. Let $\mathscr{F}^{\mathscr{M}}$ be the natural filtration generated by $X$ and suppose that there exists an intensity $\lambda$ in the sense of Definition 2.5. Note that in this example, for each $0 \leq t \leq T, Y_{t}=X_{0}, \mathscr{F}_{t}^{\mathcal{I}}$ is a trivial sigma-algebra, and so $\lambda_{t}$ is deterministic because $\lambda$ is an $\mathscr{F}^{\mathcal{I}}$-adapted process. We can determine the FS decomposition of the defaultable claim $F\left(Y_{T}\right) \tilde{N}_{T}$ (paid at $T \leq l$ for a known function $F: \mathbb{R} \rightarrow \mathbb{R})$ in $\mathscr{F}^{\mathcal{I}, \tau}$, as follows.

Since $T \leq l$ then $F\left(Y_{T}\right)=F\left(X_{0}\right)$ and the claim is $F\left(X_{0}\right) 1_{\{\tau>T\}}$. In this case, it is easy to observe that Theorem 3.1 is not useful. Nevertheless, an application of the product rule shows that $\left(h(t) 1_{\{\tau>t\}}\right)_{0 \leq t \leq T}$ is an $\mathscr{F}^{\mathcal{I}, \tau}$-square integrable martingale where $h(t)=F\left(X_{0}\right) \frac{e^{\int_{0}^{t} \lambda_{s} d s}}{e^{\int_{0}^{T} \lambda_{s} d s}}, 0 \leq t \leq T$. Therefore, we have $F\left(X_{0}\right) \widetilde{N}_{T}=\frac{F\left(X_{0}\right)}{e^{\int_{0}^{T} \lambda_{s} d s}}+L_{T}$, for an $\mathscr{F}^{\mathcal{I}, \tau}$-square integrable martingale $L$ with $L_{0}=0$. This is indeed the FS decomposition of this claim because $\left\langle L, M^{(1)}\right\rangle^{\mathscr{F}, \tau}=0$, and by Lemma 7.1 of the Appendix, $L$ is orthogonal to $M^{(1)}$.

Now, we consider an example in which managers of a firm have full access to the information, and $\tau$ is defined endogenously by asset values of the firm and hence predictable to managers but totally inaccessible to investors. Note that this example cannot be analyzed using hazard rate based models where the intensity process is modeled by Definition 2.4 .

Example 4.2. Consider a defaultable claim $(H, \tau, T)$ and suppose that $\alpha_{t}=(t-l)^{+}$, $t \geq 0$, for a deterministic constant $0<l<T, H=F\left(Y_{T}\right)$, and $b_{t}=f\left(t, X_{t}\right), t \geq 0$, for continuous functions $F: \mathbb{R} \rightarrow \mathbb{R}$ and $f:[0, \infty) \times \mathbb{R} \rightarrow[0, \infty)$.

Assume that $X$ is given by the Black-Scholes geometric Brownian motion model with parameters $\mu$ and $\sigma>0, \mathscr{F}_{t}=\mathscr{F}_{t}^{\mathscr{M}}, t \geq 0$, is the sigma-algebra generated by $\left\{W_{s} ; s \leq t\right\}$, where $W$ is a standard Brownian motion, and the default time $\tau$ is defined by $\tau=\inf \{t>$ $\left.0 ; X_{t} \leq d\right\}$, for a given barrier $0<d<X_{0}$. Then Theorem 3.1 of [15] shows that under 
$\left(\mathscr{F}_{t}^{\mathcal{I}} \vee \mathcal{N}_{t}\right)_{t \geq 0}, \lambda_{t}=g\left(Y_{t}\right), t \geq 0$, in the sense of Definition 2.5 for a continuous function $g:[0, \infty) \rightarrow[0, \infty)$. For instance, by Example 3.2 of [15], for $t<\tau$ and $t>l$, we have $\lambda_{t}=-\frac{\frac{\partial \psi}{\partial t}\left(\frac{\mu}{\sigma}-\frac{\sigma}{2}, l, \frac{\log d-\log \left(Y_{t}\right)}{\sigma}\right)}{\psi\left(\frac{\mu}{\sigma}-\frac{\sigma}{2}, l, \frac{\log d-\log \left(Y_{t}\right)}{\sigma}\right)}$, where $\psi(\theta, t, y)=1-\int_{0}^{t} \frac{|y|}{\sqrt{2 \pi s^{3}}} e^{-\frac{(y-\theta s)^{2}}{2 s}} d s$, for $y<0$.

Suppose that there is a continuous function $h:[0, T-l] \times \mathbb{R} \rightarrow \mathbb{R}$ such that $h \in$ $C^{1,2}([0, T-l) \times \mathbb{R})$, and it satisfies the PDE, $\frac{\partial h}{\partial t}(t, x)+\frac{f(t, x)}{2} \frac{\partial^{2} h}{\partial x^{2}}(t, x)-g(x) h(t, x)=0$ on $[0, T-l) \times \mathbb{R}$, with the boundary condition $h(T-l, x)=F(x)$ to match the final payoff. We recall that filtration $\left(\mathscr{F}_{t}^{\mathcal{I}} \vee \mathcal{N}_{t}\right)_{t \geq 0}$ satisfies Equation (4) and hence Definition 2.3. The FS decomposition of the defaultable claim $F\left(Y_{T}\right) \widetilde{N}_{T}$ in $\mathscr{F}^{\mathcal{I}, \tau}=\left(\mathscr{F}_{t}^{\mathcal{I}} \vee \mathcal{N}_{t}\right)_{t \geq 0}$, can be determined as follows.

Through a $C^{1,2}$ extension of $h$ to $[0, \infty) \times \mathbb{R}$ and using an analysis similar to the proof of Proposition 4.1, by part 1 of Theorem 3.1, we can show that

$$
h\left(T-l, Y_{T}\right) \widetilde{N}_{T}+\int_{0}^{T \wedge \tau \wedge l} h\left(0, X_{0}\right) \lambda_{s} d s=h\left(0, X_{0}\right)+\int_{0}^{T} \theta_{s} 1_{\{s<T\}} d Y_{s}^{\tau}+L_{T}
$$

where $\theta_{t}=\frac{\partial h}{\partial x}\left(\alpha_{t}, Y_{t}\right) 1_{\{t \leq \tau\}}, 0 \leq t<T$, and $L, L_{0}=0$, is an $\mathscr{F}^{\mathcal{I}, \tau}$-local martingale orthogonal to $M^{(1)}$. Clearly, the second term on the left-hand side of (16) becomes $h\left(0, X_{0}\right) \int_{0}^{l \wedge \tau} \lambda_{s} d s$ which is equal to $-h\left(0, X_{0}\right) \widetilde{N}_{l}+h\left(0, X_{0}\right)+L_{l}^{\prime}$ for a process $L^{\prime}, L_{0}^{\prime}=0$, which is an $\mathscr{F}^{\mathcal{I}, \tau}$-local martingale. The martingale $L^{\prime}$ is of finite variation, hence $L^{\prime}$ is orthogonal to the local martingale part of $Y^{\tau}$, i.e. $M^{(1)}$. Therefore (16) becomes

$$
h\left(T-l, Y_{T}\right) \tilde{N}_{T}-h\left(0, X_{0}\right) \tilde{N}_{l}=\int_{0}^{T} \theta_{s} 1_{\{s<T\}} d Y_{s}^{\tau}+\left(L_{T}-L_{T}^{\prime}\right) .
$$

Similar to Example 4.1, we can show that

$$
h\left(0, X_{0}\right) \widetilde{N}_{t \wedge l}=\frac{h\left(0, X_{0}\right)}{e^{\int_{0}^{l} \lambda_{s} d s}}+L_{t}^{\prime \prime}, t \geq 0,
$$

where $L^{\prime \prime}, L_{0}^{\prime \prime}=0$, is a finite variation $\mathscr{F}^{\mathcal{I}, \tau}$-local martingale orthogonal to $M^{(1)}$. By evaluating (18) at $t=T$ and adding it to (17), we have

$$
F\left(Y_{T}\right) \widetilde{N}_{T}=\frac{h\left(0, X_{0}\right)}{e^{\int_{0}^{l} \lambda_{s} d s}}+\int_{0}^{T} \theta_{s} 1_{\{s<T\}} d Y_{s}^{\tau}+\left(L_{T}-L_{T}^{\prime}+L_{T}^{\prime \prime}\right) .
$$

Note that the local martingale $L-L^{\prime}+L^{\prime \prime}$ is orthogonal to $M^{(1)}$.

Furthermore suppose that $M^{(1)}$ and $L$ are square integrable martingales, and $\theta 1_{\{\cdot<T\}}$ belongs to $\Theta$. Then the FS decomposition of the defaultable claim in $\mathscr{F}^{\mathcal{I}, \tau}$ is given by (19). 
The following example considers a hazard based model (Definition 2.4) in which $\tau$ is defined exogenously, (i.e. not defined based on the management's information), hence not adapted to neither the management's nor to the investors' filtration, and at the same time investors receive delayed data.

Example 4.3. Consider a defaultable claim $(H, \tau, T)$. Suppose that $X$ has Markov property with respect to $\mathscr{F}^{\mathscr{M}}$, and $H=F\left(Y_{T}\right)$ for a continuous function $F: \mathbb{R} \rightarrow \mathbb{R}$. For $t \geq 0$, assume that $\alpha_{t}=(t-l)^{+}$, for a deterministic constant $0<l<T, b_{t}=f\left(t, X_{t}\right)$, and $\mathbb{P}\left[\tau>t \mid \mathscr{F}_{t}^{\mathscr{M}}\right]=e^{-\int_{0}^{t} g\left(s, X_{s}\right) d s}$, for continuous functions $f:[0, \infty) \times \mathbb{R} \rightarrow[0, \infty)$ and $g:[0, \infty) \times \mathbb{R} \rightarrow[0, \infty)$. We can determine the $F S$ decomposition of $F\left(Y_{T}\right) \widetilde{N}_{T}$ in $\mathscr{F}^{\mathcal{I}, \tau}=\left(\mathscr{F}_{t}^{\mathcal{I}} \vee \mathcal{N}_{t}\right)_{t \geq 0}$ as follows.

Recall that $\mathscr{F}^{\mathcal{I}}$ is the investors' information in Assumption 2.2 given by $\mathscr{F}_{t}^{\mathcal{I}}=\mathscr{F}_{\alpha_{t}} \mathscr{M}$. Since for all $t \geq 0, \alpha_{t} \leq t$, we have $\mathscr{F}_{t}^{\mathcal{I}} \subset \mathscr{F}_{t}^{\mathscr{M}}$ and so

$$
\mathbb{P}\left[\tau>t \mid \mathscr{F}_{t}^{\mathcal{I}}\right]=e^{-\int_{0}^{\alpha_{t}} g\left(s, X_{s}\right) d s} \mathbb{E}\left[e^{-\int_{\alpha_{t}}^{t} g\left(s, X_{s}\right) d s} \mid \mathscr{F}_{t}^{\mathcal{I}}\right], t \geq 0 .
$$

Using the Markov property, the above expectation can be expressed according to a function of the triplet $\left(t, \gamma_{t}, Y_{t}\right)$, where $\gamma_{t}=t-\alpha_{t}$ and $t \geq 0$. If this function is of finite variation with respect to $t$ then we have an $\mathscr{F}^{\mathcal{I}}$-adapted process $\tilde{\lambda}$ defined by $\tilde{\lambda}_{t}=\tilde{g}\left(t, \gamma_{t}, Y_{t}\right), t \geq 0$, where $\tilde{g}$ is a function from $[0, \infty) \times[0, \infty) \times \mathbb{R}$ to $[0, \infty)$ (assumed to be continuous) such that $\mathbb{P}\left[\tau>t \mid \mathscr{F}_{t}^{\mathcal{I}}\right]=e^{-\int_{0}^{t} \tilde{\lambda}_{s} d s}$, for all $t \geq 0$.

Hence by Proposition 5.1 .3 of [6], $\left(N_{t}-\int_{0}^{t \wedge \tau} \tilde{\lambda}_{s} d s\right)_{t \geq 0}$ is a martingale in $\left(\mathscr{F}_{t}^{\mathcal{I}} \vee \mathcal{N}_{t}\right)_{t \geq 0}$. Note that on the support of the measure defined by $\alpha$, we have that $\gamma=l$ and $\tilde{\lambda}=k(\alpha, Y)$ for a continuous function $k:[0, \infty) \times \mathbb{R} \rightarrow \mathbb{R}$. Following the same steps as in Example 4.2 and an analysis similar to that of Proposition 4.1, by imposing further integrability conditions, the FS decomposition and the PLRM hedging strategy can be found by solving the PDE, $\frac{\partial h}{\partial t}(t, x)+\frac{f(t, x)}{2} \frac{\partial^{2} h}{\partial x^{2}}(t, x)-k(t, x) h(t, x)=0$ on $[0, T-l) \times \mathbb{R}$, with the boundary condition $h(T-l, x)=F(x)$, for a continuous function $h:[0, T-l] \times \mathbb{R} \rightarrow \mathbb{R}$ such that $h \in C^{1,2}([0, T-l) \times \mathbb{R})$.

The following example explains the case when $\tau$ admits an intensity process with respect to the investors' information in the sense of Definition 2.4.

Example 4.4. Consider a defaultable claim $(H, \tau, T)$ where $\tau$ may or may not be an $\mathscr{F}^{\mathscr{M}}$-stopping time. Suppose that $H=F\left(Y_{T}\right)$ for a continuous function $F: \mathbb{R} \rightarrow \mathbb{R}$. For $t \geq 0$, assume that $\alpha_{t}=(t-l)^{+}$, for a deterministic constant $0<l<T, b_{t}=f\left(t, X_{t}\right)$, and $\mathbb{P}\left[\tau>t \mid \mathscr{F}_{t}^{\mathcal{I}}\right]=e^{-\int_{0}^{t} g\left(\alpha_{s}, Y_{s}\right) d s}$, for continuous functions $f:[0, \infty) \times \mathbb{R} \rightarrow[0, \infty)$ and $g:[0, \infty) \times \mathbb{R} \rightarrow[0, \infty)$. 
Assuming the filtration expansion $\mathscr{F}^{\mathcal{I}, \tau}=\left(\mathscr{F}_{t}^{\mathcal{I}} \vee \mathcal{N}_{t}\right)_{t \geq 0}$, following the same steps as in Example 4.2, and an analysis similar to that of Proposition 4.1, by imposing further integrability conditions, the PLRM hedging strategy and the FS decomposition can be found by solving the PDE, $\frac{\partial h}{\partial t}(t, x)+\frac{f(t, x)}{2} \frac{\partial^{2} h}{\partial x^{2}}(t, x)-g(t, x) h(t, x)=0$ on $[0, T-l) \times \mathbb{R}$, with the boundary condition $h(T-l, x)=F(x)$, for a continuous function $h:[0, T-l] \times \mathbb{R} \rightarrow \mathbb{R}$ such that $h \in C^{1,2}([0, T-l) \times \mathbb{R})$.

A special case of this example is when $\tau$ is independent of the investors' information $\mathscr{F}^{\mathcal{I}}$, which leads to $\mathbb{P}\left[\tau>t \mid \mathscr{F}^{\mathcal{I}}\right]=\mathbb{P}[\tau>t], t \geq 0$. In this case, the process $Z$ (the optional projection of $\tilde{N}$ over $\mathscr{F}^{\mathcal{I}}$ ) is simply a decreasing deterministic function, and the intensity process exists in the sense of Definition 2.4, Lebesgue almost surely, given by the deterministic function $\lambda_{t}=-\frac{\partial \ln \mathbb{P}[\tau>t]}{\partial t}, t \geq 0$.

Up until now, we have applied only deterministic time changes. The following result studies the case of a stochastic time change assuming that $X$ is a local martingale.

Proposition 4.2. Consider a defaultable claim $(H, \tau, T)$ where $\tau$ may or may not be an $\mathscr{F}^{\mathscr{M}}$-stopping time. Suppose that $\mathbb{P}\left[\tau>t \mid \mathscr{F}_{t}^{\mathcal{I}}\right]=e^{-\int_{0}^{t} g\left(\alpha_{s}, Y_{s}\right) d s}, t \geq 0, H=F\left(Y_{T}\right)$, $a=0$, and $b_{t}=f\left(t, X_{t}\right), t \geq 0$, for continuous functions $g:[0, \infty) \times \mathbb{R} \rightarrow[0, \infty)$, $F: \mathbb{R} \rightarrow \mathbb{R}$, and $f:[0, \infty) \times \mathbb{R} \rightarrow[0, \infty)$. Assume that there is a continuous function $h:[0, T] \times \mathbb{R} \rightarrow \mathbb{R}$, such that $h \in C^{1,2}([0, T) \times \mathbb{R})$, and $h$ is a solution of the following $P D E$ :

$$
\begin{aligned}
& \frac{\partial h}{\partial t}(t, x)+\frac{1}{2} \frac{\partial^{2} h}{\partial x^{2}}(t, x) f(t, x)-g(t, x) h(t, x)=0,(t, x) \in[0, T) \times \mathbb{R}, \\
& h(T, x)=F(x) .
\end{aligned}
$$

We assume that $\alpha_{T}=T$, and $\gamma$ is absolutely continuous (hence so is $\alpha$ ) with respect to the Lebesgue measure with the Radon-Nikodym derivative $\beta_{t}=\frac{d \gamma_{t}}{d t}, t \geq 0$, such that $h\left(\alpha_{\tau}, Y_{\tau}\right) \beta_{\tau}$ is integrable. Let $F_{t}=\mathbb{P}\left(\tau \leq t \mid \mathscr{F}_{t}^{\mathcal{I}}\right), t \geq 0$. If the process $m$ defined by $m_{t}=\mathbb{E}\left[\int_{0}^{T} h\left(\alpha_{u}, Y_{u}\right) \beta_{u} d F_{u} \mid \mathscr{F}_{t}^{\mathcal{I}}\right], t \geq 0$, is continuous at $\tau$, and $\mathscr{F}^{\mathcal{I}, \tau}=\left(\mathscr{F}_{t}^{\mathcal{I}} \vee \mathcal{N}_{t}\right)_{t \geq 0}$, then

$$
H \tilde{N}_{T}=h\left(0, X_{0}\right)-m_{0}+\int_{0}^{T}\left(\theta_{t} 1_{\{t<T\}}-\theta_{t}^{*}\right) d Y_{t}^{\tau}+L_{T}
$$

where for $0 \leq t \leq T, \theta_{t}^{*}=e^{\Gamma_{t}} \frac{d\left[\tilde{m}, Y^{\tau}\right]_{t}}{d\left[Y^{\tau}\right]_{t}}, \Gamma_{t}=\int_{0}^{t} g\left(\alpha_{s}, Y_{s}\right) d s, \tilde{m}_{t}=m_{t \wedge \tau}, \theta_{t}=$ $\frac{\partial h}{\partial x}\left(t, Y_{t}\right) 1_{\{t \leq \tau\}}$ (excluding the case $t=T$ ), and $L=\left(L_{t}\right)_{t \geq 0}, L_{0}=0$, is an $\mathscr{F}^{\mathcal{I}, \tau}$-local martingale orthogonal to $Y^{\tau}$.

Furthermore, suppose that $M^{(1)}$ and $L$ are square integrable martingales, and $\theta 1_{\{\cdot<T\}}$ and $\theta^{*}$ belong to $\Theta$. Then the FS decomposition of the defaultable claim is given by $(20)$. 
Proof. Since $a=0, Y$ is an $\mathscr{F}^{\mathcal{I}}$-local martingale, by Theorem 7.1 of the Appendix, then $Y^{\tau}$ is an $\mathscr{F}^{\mathcal{I}, \tau}$-local martingale, and so LRM reduces to risk-minimization.

Through a $C^{1,2}$ extension of $h$ to $[0, \infty) \times \mathbb{R}$ and using an analysis similar to the proof of Proposition 4.1, by part 1 of Theorem 3.1, we can show that

$$
h\left(\alpha_{T}, Y_{T}\right) \widetilde{N}_{T}+\int_{0}^{T \wedge \tau} h\left(\alpha_{s}, Y_{s}\right) \lambda_{s} d \gamma_{s}=h\left(0, X_{0}\right)+\int_{0}^{T} \theta_{s} 1_{\{s<T\}} d Y_{s}^{\tau}+L_{T}^{(1)},
$$

where $L^{(1)}, L_{0}^{(1)}=0$, is an $\mathscr{F}^{\mathcal{I}, \tau}$-local martingale orthogonal to $Y^{\tau}$. Because $\left(N_{t}-\right.$ $\left.\int_{0}^{t \wedge \tau} \lambda_{s} d s\right)_{t \geq 0}$ is an $\mathscr{F}^{\mathcal{I}, \tau}$-martingale, the integral on the left-hand side of (21), can be written as

$$
\int_{0}^{T \wedge \tau} h\left(\alpha_{s}, Y_{s}\right) \lambda_{s} d \gamma_{s}=\int_{0}^{T} h\left(\alpha_{s}, Y_{s}\right) \beta_{s} d N_{s}+L_{T}^{(2)}=h\left(\alpha_{\tau}, Y_{\tau}\right) \beta_{\tau} N_{T}+L_{T}^{(2)},
$$

where $L^{(2)}, L_{0}^{(2)}=0$, is an $\mathscr{F}^{\mathcal{I}, \tau}$-local martingale.

By Proposition 5.2.1 of [6], we have

$$
\mathbb{E}\left[h\left(\alpha_{\tau}, Y_{\tau}\right) \beta_{\tau} N_{T} \mid \mathscr{F}_{t}^{\mathcal{I}, \tau}\right]=m_{0}+\int_{0}^{t} e^{\Gamma_{u}} d \tilde{m}_{u}+L_{t}^{(3)}, t \geq 0
$$

where for $t \geq 0, \tilde{m}_{t}=m_{t \wedge \tau}, m_{t}=\mathbb{E}\left[\int_{0}^{T} h\left(\alpha_{u}, Y_{u}\right) \beta_{u} d F_{u} \mid \mathscr{F}_{t}^{\mathcal{I}}\right], \Gamma_{t}=-\ln \left(\mathbb{P}\left(\tau>t \mid \mathscr{F}_{t}^{\mathcal{I}}\right)\right)=$ $\int_{0}^{t} g\left(\alpha_{s}, Y_{s}\right) d s$ as in Definition 2.4, and $L^{(3)}, L_{0}^{(3)}=0$, is an $\mathscr{F}^{\mathcal{I}, \tau}$-local martingale of finite variation. This leads to $h\left(\alpha_{\tau}, Y_{\tau}\right) \beta_{\tau} N_{T}=m_{0}+\int_{0}^{T} e^{\Gamma_{u}} d \tilde{m}_{u}+L_{T}^{(3)}$.

By Theorem 7.1 of the Appendix, the process $\tilde{m}$ is an $\mathscr{F}^{\mathcal{I}, \tau}$-local martingale and so is $\left(\int_{0}^{t} e^{\Gamma_{u}} d \tilde{m}_{u}\right)_{t \geq 0}$. Therefore, $\left(\int_{0}^{t} e^{\Gamma_{u}} d \tilde{m}_{u}\right)_{t \geq 0}$ admits a $\mathrm{KW}$ decomposition versus $Y^{\tau}$ in $\mathscr{F}^{\mathcal{I}, \tau}$, i.e. $\int_{0}^{t} e^{\Gamma_{u}} d \tilde{m}_{u}=\int_{0}^{t} \theta_{u}^{*} d Y_{u}^{\tau}+L_{t}^{(4)}, t \geq 0$, where $\theta^{*}$ is given by $\theta_{t}^{*}=e^{\Gamma_{t}} \frac{d\left[\tilde{m}, Y^{\tau}\right]_{t}}{d\left[Y^{\tau}\right]_{t}}$, $t \geq 0$, and $L^{(4)}, L_{0}^{(4)}=0$, is an $\mathscr{F}^{\mathcal{I}, \tau}$-local martingale orthogonal to $Y^{\tau}$.

So we have $h\left(\alpha_{\tau}, Y_{\tau}\right) \beta_{\tau} N_{T}=m_{0}+\int_{0}^{T} \theta_{s}^{*} d Y_{s}^{\tau}+L_{T}^{(4)}+L_{T}^{(3)}$; this together with (21) and (22) lead to the following equation:

$$
\begin{aligned}
h\left(\alpha_{T}, Y_{T}\right) \tilde{N}_{T} & =h\left(T, Y_{T}\right) \tilde{N}_{T} \\
& =h\left(0, X_{0}\right)-m_{0}+\int_{0}^{T}\left(\theta_{t} 1_{\{t<T\}}-\theta_{t}^{*}\right) d Y_{t}^{\tau}+L_{T}^{(1)}-L_{T}^{(2)}-L_{T}^{(3)}-L_{T}^{(4)} .
\end{aligned}
$$

We have already observed that the $\mathscr{F}^{\mathcal{I}, \tau}$-local martingales $L^{(1)}$ and $L^{(4)}$ are orthogonal to $Y^{\tau}$. On the other hand, $L^{(2)}$ and $L^{(3)}$ are finite variation $\mathscr{F}^{\mathcal{I}, \tau}$-local martingales and hence orthogonal to $Y^{\tau}$ (by Lemma 7.1 of the Appendix). Therefore, $L$ defined by $L=L^{(1)}-L^{(2)}-L^{(3)}-L^{(4)}$ is orthogonal to $Y^{\tau}$ which proves the result. 
Remark 4.2. One of the challenges of applying a random time change within the setup of the previous proposition, is to have random time changes that satisfy $\alpha_{T}=T$ so that $h\left(\alpha_{T}, Y_{T}\right)=F\left(Y_{T}\right)$. The condition $\alpha_{T}=T$ is a technical assumption to avoid random boundary conditions for the PDEs. However, it can be also financially interpreted as a maturity time that lies on a date when the company's accounting data are released so that the investors would have access to almost the same level of information as the management.

As an example of such time change, consider a random time $T_{1}$ such that $0<T_{1}<T$ a.s., and for each $t \geq 0, \alpha_{t}=\frac{T\left(t-T_{1}\right)}{T-T_{1}} 1_{\left\{t>T_{1}\right\}}$ is an $\mathscr{F}^{\mathscr{M}}$-stopping time. Therefore, $\left(\alpha_{t}\right)_{t \geq 0}$ defines a random time change based on Definition 2.1. This random time change represents scenarios in which there is no release of information up until time $T_{1}$ and then thereafter the data is getting updated gradually until $T$.

\section{Conclusion}

In this paper, we have discussed a partial information credit risk model where the solutions of hedging strategies of defaultable claims are given through the PLRM approach assuming that the information is received with some delay, and the delay is modeled by a random time change. In doing so, two notions of intensity are applied which allow for the default event to be defined both endogenously and exogenously for example as the first hitting time of underlying assets to a barrier. Under some conditions, the numerical implementation can be executed through the solution of PDEs.

\section{Acknowledgments}

The author is very thankful to Thorsten Rheinländer, Helen Ogden, and two anonymous referees for many useful comments and suggestions.

\section{References}

[1] Ansel, J.P. and Stricker, C., 1993. Décomposition de Kunita-Watanabe. Séminaire de probabilités de Strasbourg, 27, 30-32.

[2] Aven, T., 1985. A theorem for determining the compensator of a counting process. Scand. J. Statist., 12, 69-72. 
[3] Biagini, F. and Cretarola, A., 2007. Quadratic hedging methods for defaultable claims. Appl. Math. Optim., 56 (3), 425-443.

[4] Biagini, F. and Cretarola, A., 2009. Local risk-minimization for defaultable markets. Math. Finance, 19 (4), 669-689.

[5] Biagini, F. and Cretarola, A., 2012. Local risk-minimization for defaultable claims with recovery process. Appl Math Optim., 65 (3), 293-314.

[6] Bielecki, T.R. and Rutkowski, M., 2004. Credit Risk: modelling, valuation and hedging. Springer: Berlin.

[7] Björk, T., 2009. Arbitrage theory in continuous time. Oxford university press.

[8] Ceci, C., Cretarola, A. and Colaneri, K., 2015. Local risk-minimization under restricted information on asset prices. Electron. J. Probab., 20 (96), 1-30.

[9] Ceci, C., Cretarola, A. and Colaneri, K., 2017. The Föllmer-Schweizer decomposition under incomplete information. Stochastics, 89 (8), 1166-1200

[10] Ceci, C., Cretarola, A. and Russo, F., 2014. BSDEs under partial information and financial applications. Stoch. Proc. and their Appl., 124 (8), 2628-2653.

[11] Choulli, T., Vandaele, N. and Vanmaele, M., 2010. The Föllmer-Schweizer decomposition: Comparison and description. Stoch. Proc. and their Appl., 120 (6), 853-872.

[12] Dellacherie, C. and Meyer, P., 1982. Probabilities and Potential B, volume 72 of North-Holland Mathematics Studies. North-Holland Publishing Co., Amsterdam.

[13] Föllmer, H. and Schweizer, M., 1991. Hedging of contingent claims under incomplete information. Applied Stochastic Analysis, 5, 389-414.

[14] Giesecke, K., 2006. Default and information. J. Econom. Dynam. Control, 30 (11), $2281-2303$.

[15] Guo, X., Jarrow, R.A. and Zeng, Y., 2009. Credit risk models with incomplete information. Mathematics of Operations Research, 34 (2), 320-332.

[16] Guo, X. and Zeng, Y., 2008. Intensity process and compensator: A new filtration expansion approach and the Jeulin-Yor theorem. The Annals of Applied Probability, 18 (1), 120-142. 
[17] He, S.W. and Yan, J.A., 1992. Semimartingale theory and stochastic calculus. Taylor \& Francis.

[18] Jacod, J. and Shiryaev, A.N., 2003. Limit theorems for stochastic processes. Springer Science \& Business Media.

[19] Jeanblanc, M. and Le Cam, Y., 2009. Progressive enlargement of filtrations with initial times. Stochastic Processes and their Applications, 119 (8), 2523-2543.

[20] Jeulin, T. and Yor, M., 1985. Grossissements de filtrations: exemples et applications. Volume 1118 of Lecture Notes in Mathematics. Springer, Berlin.

[21] Kallsen, J. and Shiryaev, A. N., 2002. Time change representation of stochastic integrals. Theory of Probability 83 Its Applications, 46 (3), 522-528.

[22] Karatzas, I. and Shreve, S., 1991. Brownian motion and stochastic calculus. SpringerVerlag, Berline Heidelberg New York.

[23] Kchia, Y., Larsson M. and Protter P., 2013. Linking progressive and initial filtration expansions. In: Viens F., Feng J., Hu Y., Nualart E. (eds) Malliavin Calculus and Stochastic Analysis. Springer Proceedings in Mathematics 6 Statistics, 34. Springer, Boston, MA.

[24] Okhrati, R., Balbas, A. and Garrido, J., 2014. Hedging of defaultable claims in a structural model using a locally risk-minimizing approach. Stochastic Processes and their Applications, 124 (9), 2868-2891.

[25] Protter, P., 2004. Stochastic integration and differential equations. Springer-Verlag: Berlin.

[26] Revuz, D. and Yor, M., 2005. Continuous martingales and Brownian motion (Vol. 293, Third Edition). Springer Science \& Business Media.

[27] Schweizer, M., 1995, On the minimal martingale measure and the Föllmer-Schweizer decomposition, Stochastic Analysis and Applications, 13 (5), 573-599.

[28] Schweizer, M., 2001. A guided tour through quadratic hedging approaches, in Handbook in Mathematical Finance: Option Pricing, Interest Rates and Risk Management, E. Jouini, J. Cvitanić and M. Musiela (eds.). Cambridge University Press, Cambridge, 538-574. 
[29] Whitney, H., 1934. Analytic extensions of differentiable functions defined in closed sets. Trans. Amer. Math. Soc., 36 (1), 63-89.

\section{Appendix}

\subsection{Definitions and Technical Results}

For the convenience of the reader, we recall certain basic notation and definitions. We suppose that $(\Omega, \mathfrak{F}, \mathcal{F}, \mathbb{P})$ is a complete probability space equipped with a filtration $\mathcal{F}=$ $\left(\mathcal{F}_{t}\right)_{t \geq 0}$ which satisfies the usual hypotheses and $\mathcal{F}_{t} \subset \mathfrak{F}$ for all $t \geq 0$. Let $\mathcal{F}_{\infty}$ be the sigma-algebra generated by all $\mathcal{F}_{t}, t \geq 0$.

Definition 7.1. Suppose that $U$ and $R$ are two semimartingales in $(\Omega, \mathfrak{F}, \mathcal{F}, \mathbb{P})$, then

- The quadratic covariation of $U$ and $R$ is denoted by $[U, R]$ and defined by $[U, R]=$ $U R-U_{0} R_{0}-\int U_{-} d R-\int R_{-} d U$.

- The predictable quadratic covariation of $U$ and $R$ is denoted by $\langle U, R\rangle^{\mathcal{F}}$ and defined to be an $\mathcal{F}$-predictable process with locally of integrable variation (see Definitions I.3.7 and I.3.8 of [18]) null at time zero (if such a process exists in the first place) such that $[U, R]-\langle U, R\rangle^{\mathcal{F}}$ is an $\mathcal{F}$-local martingale.

All the important properties of (predictable) quadratic covariation can be found in Theorem I.4.47, Proposition I.4.49, and Proposition I.4.50 of [18]. In the following remark, we highlight a few important ones.

Remark 7.1. The quadratic covariation enjoys several nice properties: as a map from $(U, R)$ to $[U, R]$, it is bi-linear and symmetric. If $U$ is a semimartingale, and $R$ is of finite variation, then $[U, R]=0$ whenever either $U$ or $R$ is continuous. Furthermore, $\Delta[U, R]=\Delta U \Delta R$, which concludes that $[U, R]$ is a continuous process (hence predictable and by Lemma I.3.10 of [18] with locally of integrable variation) whenever either one of $U$ or $R$ is continuous.

The superscript $\mathcal{F}$ in $\langle U, R\rangle^{\mathcal{F}}$ indicates the relevance to the filtration $\mathcal{F}$, and it can be dropped if the reference filtration is obvious. Note that if $\langle U, R\rangle^{\mathcal{F}}$ exists, it is uniquely determined by Corollary I.3.16 of [18] up to an evanescent set. Also, if $[U, R]$ belongs to the class of locally integrable variation processes or if $U$ and $R$ are locally square integrable martingales, Theorem I.3.18 and Proposition I.4.50 of [18] guarantee the existence of $\langle U, R\rangle^{\mathcal{F}}$. In fact, the predictable quadratic covariation is originally defined for locally 
square integrable martingales (see Theorem I.4.2 of [18]), here we have slightly generalized this by Definition 7.1.

As mentioned earlier, if either one of $U$ or $R$ is continuous, then $[U, R]$ is predictable with locally of integrable variation. Therefore, $[U, R]=\langle U, R\rangle^{\mathcal{F}}$ by the uniqueness of predictable quadratic covariation, and the superscript $\mathcal{F}$ could be dropped.

Remark 7.2. Suppose that $U$ and $R$ are two semimartingales, by Theorem 29, Chapter 2 of [25], for any càglàd processes $K^{(1)}$ and $K^{(2)}$, we have

$$
\left[\int K^{(1)} d U, \int K^{(2)} d R\right]=\int K^{(1)} K^{(2)} d[U, R]
$$

and if $\left\langle\int K^{(1)} d U, \int K^{(2)} d R\right\rangle^{\mathcal{F}}$ and $\int K^{(1)} K^{(2)} d\langle U, R\rangle^{\mathcal{F}}$ exist according to Definition \%.1, then the uniqueness of the predictable quadratic covariation shows that

$$
\left\langle\int K^{(1)} d U, \int K^{(2)} d R\right\rangle^{\mathcal{F}}=\int K^{(1)} K^{(2)} d\langle U, R\rangle^{\mathcal{F}} .
$$

Definition 7.2. Two local martingales $\mathfrak{M}$ and $\mathfrak{N}$ are called orthogonal if $\mathfrak{M N}$ is again a local martingale.

Lemma 7.1. Suppose that $\mathfrak{M}$ and $\mathfrak{N}$ are two local martingales such that $\langle\mathfrak{M}, \mathfrak{N}\rangle^{\mathcal{F}}$ exists based on the second part of Definition 7.1. Then $\mathfrak{M}$ and $\mathfrak{N}$ are orthogonal if and only if $\langle\mathfrak{M}, \mathfrak{N}\rangle^{\mathcal{F}}=0$.

Proof. From the definition of quadratic covariation, the orthogonality of $\mathfrak{M}$ and $\mathfrak{N}$ is equivalent to $[\mathfrak{M}, \mathfrak{N}]$ being a local martingales. Also, by the definition of predictable quadratic covariation, $[\mathfrak{M}, \mathfrak{N}]-\langle\mathfrak{M}, \mathfrak{N}\rangle^{\mathcal{F}}$ is a local martingale. Therefore, the orthogonality of $\mathfrak{M}$ and $\mathfrak{N}$ is equivalent to $\langle\mathfrak{M}, \mathfrak{N}\rangle^{\mathcal{F}}$ being a local martingale. Because $\langle\mathfrak{M}, \mathfrak{N}\rangle^{\mathcal{F}}$ is a predictable finite variation local martingale, Corollary I.3.16 of [18] shows that $\langle\mathfrak{M}, \mathfrak{N}\rangle^{\mathcal{F}}=0$.

Remark 7.3. As we discussed earlier, if $\mathfrak{M}$ and $\mathfrak{N}$ are two locally square integrable martingales, then $\langle\mathfrak{M}, \mathfrak{N}\rangle^{\mathcal{F}}$ exists. This means that two locally square integrable martingales are orthogonal if and only if their predictable quadratic covariation is zero.

The following result is a special case of Theorem 2 of [23] (see also [19]), and it is used to provide the canonical decomposition of a locally square integrable martingale stopped at a random time in expanded filtrations. Suppose that the process $Z^{\mathcal{F}}$ is the optional projection of the process $\left(1_{\{\mathcal{T}>t\}}\right)_{t \geq 0}$ over the filtration $\mathcal{F}$. Note that $Z^{\mathcal{F}}$ is a special semimartingale and admits a canonical decomposition. 
Theorem 7.1. Suppose that $\mathfrak{M}$ is a locally square integrable martingale on $(\Omega, \mathfrak{F}, \mathcal{F}, \mathbb{P})$ and $\mathcal{T}$ is a $\mathbb{P}$-a.s. positive random variable on this space such that $\Delta \mathfrak{M}_{\mathcal{T}}=0$. Let $\mathfrak{M}^{\left(Z^{\mathcal{F}}\right)}$ be the martingale part of the canonical decomposition of $Z^{\mathcal{F}}$ in $\mathcal{F}$.

Then the process

$$
\mathfrak{M}^{\mathcal{T}}-\int \frac{1}{Z_{-}^{\mathcal{F}}} 1_{\{\cdot \leq \mathcal{T}\}} d\left\langle\mathfrak{M}_{,} \mathfrak{M}^{\left(Z^{\mathcal{F}}\right)}\right\rangle^{\mathcal{F}}
$$

is a local martingale in any filtration expansion $\mathcal{F}^{\mathcal{T}}$ of $\mathcal{F}$ by $\mathcal{T}$ that turns $\mathcal{T}$ into a stopping time and satisfies

$$
\mathcal{F}_{t}^{\mathcal{T}} \cap\{\mathcal{T}>t\}=\mathcal{F}_{t} \cap\{\mathcal{T}>t\}
$$

Definition 7.3. Assume that processes $\mathfrak{M}$ and $\mathfrak{N}$ are two local martingales. Then we say that $\mathfrak{N}$ admits a $K W$ decomposition versus $\mathfrak{M}$ if there is a predictable process $\xi$ such that the process $\int \xi^{2} d\langle\mathfrak{M}\rangle^{\mathcal{F}}$ is locally integrable and the following decomposition holds:

$$
\mathfrak{N}=\mathfrak{N}_{0}+\int \xi d \mathfrak{M}+L,
$$

where $L$ with $L_{0}=0$, is a local martingale orthogonal to $\mathfrak{M}$.

Remark 7.4. In [1], $K W$ decomposition is discussed under four cases. In general, its existence is not guaranteed, however, if $\mathfrak{N}$ and $\mathfrak{M}$ are locally square integrable martingale or when $\mathfrak{M}$ is continuous, this decomposition exists. We refer to the aforementioned reference for further discussions. 